\title{
AdS-phobia, the WGC, the Standard Model and Supersymmetry
}

\author{
Eduardo Gonzalo, Alvaro Herráez and Luis E. Ibáñez \\ Departamento de Física Teórica and Instituto de Física Teórica UAM/CSIC, \\ Universidad Autónoma de Madrid, \\ Cantoblanco, 28049 Madrid, Spain \\ E-mail: eduardo.gonzalo@uam.es, alvaro.herraez@uam.es, \\ luis.ibannez@uam.es
}

ABSTRACT: It has been recently argued that an embedding of the SM into a consistent theory of quantum gravity may imply important constraints on the mass of the lightest neutrino and the cosmological constant $\Lambda_{4}$. The constraints come from imposing the absence of any non-SUSY AdS stable vacua obtained from any consistent compactification of the SM to 3 or 2 dimensions. This condition comes as a corollary of a recent extension of the Weak Gravity Conjecture (WGC) by Ooguri and Vafa. In this paper we study $T^{2} / Z_{N}$ compactifications of the SM to two dimensions in which SM Wilson lines are projected out, leading to a considerable simplification. We analyze in detail a $T^{2} / Z_{4}$ compactification of the SM in which both complex structure and Wilson line scalars are fixed and the potential is only a function of the area of the torus $a^{2}$. We find that the SM is not robust against the appearance of AdS vacua in 2D and hence would be by itself inconsistent with quantum gravity. On the contrary, if the SM is embedded at some scale $M_{S S}$ into a SUSY version like the MSSM, the AdS vacua present in the non-SUSY case disappear or become unstable. This means that WGC arguments favor a SUSY version of the SM, independently of the usual hierarchy problem arguments. In a $T^{2} / Z_{4}$ compactification in which the orbifold action is embedded into the $B-L$ symmetry the bounds on neutrino masses and the cosmological constant are recovered. This suggests that the MSSM should be extended with a $\mathrm{U}(1)_{B-L}$ gauge group. In other families of vacua the spectrum of SUSY particles is further constrained in order to avoid the appearance of new AdS vacua or instabilities. We discuss a possible understanding of the little hierarchy problem in this context.

KEYwords: Compactification and String Models, Superstring Vacua, Supersymmetric Standard Model

ArXiv EPrint: 1803.08455 


\section{Contents}

1 Introduction 1

2 The $\mathrm{SM}$ in 3D 5

2.1 The SM on the circle I $\left(R \gtrsim 1 / m_{e}\right) \quad 5$

2.2 The SM on the circle II $\left(1 / m_{e} \gtrsim R \gtrsim 1 / M_{\mathrm{EW}}\right) \quad 9$

2.3 Fixing the Wilson lines. The segment $S^{1} / Z_{2}$

3 The SM on the $T^{2} / Z_{4}$ orbifold $\quad 16$

3.1 The SM on the $T^{2}$ torus 16

$\begin{array}{ll}3.2 \text { SM compactification on the } T^{2} / Z_{4} \text { orbifold } & 19\end{array}$

3.3 Embedding $Z_{4}$ into discrete gauge symmetries 22

3.3.1 AdS SM $Z_{4}$ vacua 22

$4 \quad Z_{4}$ MSSM vacua $\quad 23$

4.1 Embedding into a discrete subgroup of $\mathrm{U}(1)_{B-L}$

4.2 Embedding into the U(1) $)_{s}$ R-symmetry 25

4.2.1 New AdS vacua for particular choices of SUSY spectra 28

$\begin{array}{ll}\text { 4.2.2 Charge-colour breaking AdS minima } & 29\end{array}$

4.3 Twisted sectors 31

5 Conclusions and outlook $\quad 32$

A One-loop effective potential in a $S^{1}$ compactification of the Standard Model

B One-loop effective potential in $T^{2} / Z_{4} \quad 38$

C Consistency of the expansion about flat background in the non-compact $\begin{array}{ll}\text { dimensions } & 41\end{array}$

D Example of MSSM spectrum $\quad 43$

\section{Introduction}

In this paper we study possible constraints on Standard Model (SM) physics derived from its eventual embedding into a consistent theory of quantum gravity. As first emphasised by Vafa [1], many simple field theories do not admit such an embedding and populate what he called the swampland of theories. Since then there has been an important effort in trying to delimitate what is the space of theories which belong to the swampland, see [2-31] for an incomplete list of references and [32] for a recent review. The most studied constraints to delimit the swampland are those given by the Weak Gravity Conjecture (WGC) [2, 3], 
which loosely speaking implies that the gravitational interaction must be weaker than gauge interactions in any consistent theory of quantum gravity. In its simplest incarnation of a $\mathrm{U}(1)$ theory coupled to gravity it implies that there must be at least one charged particle with mass $m$ and charge $Q$ such that $m \leq Q$ in Planck units, and is motivated by blackhole physics. There are different versions of the conjecture and also extensions to multiple U(1)'s and to the antisymmetric gauge tensors of string theory and supergravity, see [2-32]. In the latter case it is the tension of the branes of string theory which are bounded in terms of the charge of the antisymmetric RR or NS tensors, so that $T \leq Q$. Recently Ooguri and Vafa (OV) put forward a sharpened version of the WGC [33] (see also [34-37]) stating that in non-SUSY theories the inequality is strict, $T<Q$. When applied to string flux vacua the OV conjecture implies that all consistent AdS vacua must be either SUSY or unstable. This instability implies that there is no CFT dual and, in this sense, there is no consistent quantum gravity theory with a stable non-SUSY AdS vacuum. Indeed there is so far no counterexample to this conjecture in string theory. ${ }^{1}$ The authors of [33] go also beyond the string theory setting and conjecture that non-SUSY AdS/CFT duality is in the swampland in general, and not only for flux vacua.

We will call AdS-phobia for short the condition of the absence of any AdS stable nonSUSY vacuum in a given theory, as suggested by the more general OV condition. AdSphobia, if correct, would be a very powerful constraint on physics models. For example it would drastically reduce the possibilities in the string landscape: only Minkowski and de Sitter vacua would be consistent with quantum gravity and only those (in addition to SUSY ones) would in principle count in addressing the enormous multiplicity of vacua in string theory.

It has been recently pointed out that AdS-phobia, if applied to the SM, would have important implications on SM physics [33, 39, 40]. The point is that, as described in [41], compactifying the SM to 3 or 2 dimensions one may obtain AdS vacua, depending on the values of the cosmological constant $\Lambda_{4}$ and the neutrino masses. On the other hand the assumption of background independence implies that if a theory is consistent in $4 \mathrm{D}$, all of its compactifications should be consistent also, since we have only changed the background geometry. Thus the existence of these lower dimensional AdS vacua (if stable) would imply the inconsistency of the SM itself [33, 39]. A detailed analysis of these constraints on neutrino masses and the cosmological constant was presented in [39]. Four interesting conclusions were obtained by imposing the absence of AdS minima,

- Majorana neutrinos (two degrees of freedom per neutrino) are not possible [33].

- The lightest neutrino mass is bounded as $m_{\nu_{1}} \leq 4 \times 10^{-3} \mathrm{eV}$ (normal hierarchy) or $m_{\nu_{3}} \leq 1 \times 10^{-3} \mathrm{eV}$ (inverted hierarchy) [39].

- The cosmological constant $\Lambda_{4}$ is bounded from below by the mass of the lightest (Dirac) neutrino, $\Lambda_{4} \gtrsim m_{\nu_{i}}^{4}[39]$.

\footnotetext{
${ }^{1}$ There are examples of non-SUSY theories with AdS/CFT duals but, unlike the SM coupled to gravity, there are infinite towers of higher spin particles and do not correspond to ordinary Einstein gravity with a finite number of fields, see e.g. [38] and references therein).
} 
- The upper bound on the lightest neutrino mass implies an upper bound on the Electro-Weak scale (for fixed neutrino Yukawa couplings) [39, 40].

The last point is obvious from the fact that the neutrino mass depends on the Higgs vev. Any increase in the EW scale above a scale $\simeq 1 \mathrm{TeV}$ would make the lightest neutrino mass to be too large and violate the bound to avoid AdS. This is remarkable because it would imply that values of the EW scale much above the observed scale would belong to the swampland. Thus, in this sense, the EW fine-tuning problem would be a mirage, since the EW scale is fixed in order to avoid AdS vacua, and its scale is secretly dictated by $\Lambda_{4}$ and the neutrino Yukawa couplings. The smallness of the EW scale compared to the Planck scale would be related to the even greater hierarchy of the cosmological constant versus the Planck scale [39, 40].

In [39] the neutrino AdS vacua in $3 \mathrm{D}$ and $2 \mathrm{D}$ were analyzed in detail. These minima appear from an interplay between the (positive) dimensional reduction of the cosmological constant term, the (negative) Casimir contribution of the photon and graviton and the (positive) Casimir contribution of the lightest fermions of the SM, the neutrinos. If the lightest neutrino is sufficiently light, its contribution can destroy the AdS vacuum and make the SM AdS-safe. This is the origin of the bound on the lightest neutrino. The Casimir contribution of the other SM particles is exponentially suppressed with their mass. However as one explores the radion potential (e.g. in $3 \mathrm{D}$ in a circle) at shorter radius $\mathrm{R}$, the thresholds of the other SM particles are reached and their contribution needs to be taken into account. So the question arises whether there are further local minima or runaway directions at shorter radii. In particular, as one goes up in energies (smaller $R$ ) additional scalar moduli appear associated first with the Wilson line of the photon and then, above the hadron threshold, two Wilson lines in the Cartan subalgebra of QCD. At even shorter distances the Wilson line of the $Z^{0}$ appears. So the full moduli space in $3 \mathrm{D}$ involves one radion and four Wilson lines, with a scalar potential depending on all of them. An analysis of the existence of these additional Wilson line dependent minima was recently carried out in [42], concluding that indeed runaway field directions at smaller $\mathrm{R}$ exist for non-trivial photon Wilson line. The $R \simeq 1 / m_{\nu}$ AdS vacua are bona-fide local minima but if they could tunnel into these field directions there would be no constraints on neutrino masses nor the EW hierarchy.

In this paper we reexamine and extend the study of the AdS minima of the SM both to smaller compact radii and geometries other than the circle and the torus. It is useful to classify the vacua we find in three categories:

- Type D (Dangerous). Vacua which contain a stable AdS minimum which cannot be avoided by constraining free parameters of the theory.

- Type $S$ (Safe). Vacua which contain no stable AdS minima.

- Type P (Predictive). Vacua which contain no stable AdS minima for a certain range of the free parameters of the theory.

Our attitude concerning instability will be conservative in the sense that we will consider unstable any potential which includes any runaway direction in the fields, even though we 
are not certain how the tunneling from local minima to those runaway directions could take place in detail. We find minima in the three categories. After examining the case of the compactification on a circle, we discuss SM compactifications in which Wilson line moduli are fixed. After a brief discussion of the compactification on the segment $S^{1} / Z_{2}$, we discuss the compactification on toroidal orbifolds $T^{2} / Z_{N}$. We specialize to the case of the $T^{2} / Z_{4}$ orbifold, but most implications apply to $Z_{N}, N=2-6$ in general. The reason to study this particular background is twofold. On the one hand, unlike the case of the parity reflection in the segment, a $Z_{4}$ rotation is a symmetry of the uncompactified $4 \mathrm{D}$ SM. Secondly, in such a background all the Wilson lines as well as the complex structure are fixed, so that the scalar potential depends only on the torus area. This simplifies enormously the study of the scalar potential at shorter distances and runaway directions induced by Wilson line moduli disappear. It turns out that, in order to obtain predictive $T^{2} / Z_{4}$ vacua one needs to embed the discrete rotation into internal symmetries of the SM. We find that different embeddings into the SM gauge group lead necessarily to stable AdS vacua, rendering the SM inconsistent with quantum gravity. This is true irrespective of the value of neutrino masses or any other free particle physics parameter. Thus some of the SM vacua are of Type $D$ and make the SM as such not viable.

The underlying reason why the SM has these AdS vacua is that it is too fermionic, it has many more fermions than bosons. The dangerous AdS vacua could become unstable Type $S$ if there were enough bosons to compensate in the Casimir potential. A situation in which this naturally happens is a SUSY embedding of the SM like the MSSM. Thus, unlike the SM, the MSSM seems robust against the generation of stable AdS vacua to the extent that we have not found vacua of Type $D$. Furthermore we find $Z_{4}$ embeddings into a discrete subgroup of the $\mathrm{U}(1)_{B-L}$ gauge symmetry leading to AdS vacua of Type $P$ which may be evaded for appropriate neutrino masses, like the original $3 \mathrm{D}$ vacua in the circle, and hence are predictive. This suggests that the MSSM should be extended at some scale with an extra $\mathrm{U}(1)_{B-L}$ gauge symmetry. Another class of embeddings into discrete R-symmetries, in addition to neutrinos, also constraint the masses of the SUSY particles in a way essentially dictated by the sign of the supertrace $\delta_{S S}=\sum_{i}(-1)^{\left(n_{i}\right)} m_{i}^{2}$. More generally, in SUSY models avoiding AdS vacua from charge-colour breaking AdS minima inherited from the 4D potential impose additional constraints. Thus an AdS-safe MSSM should pass both these constraints, leading potentially to interesting conditions for SUSY model building.

As discussed in [39, 40], avoiding the AdS neutrino vacua imply an upper bound on the EW scale close to its experimental value. On the other hand absence of lower $4 \mathrm{D}$ chargecolour breaking minima requires typically a relatively massive SUSY spectrum. This could provide for an explanation for the little hierarchy problem of the MSSM: absence of AdS neutrino vacua requires a EW scale close to its experimental value, while absence of chargecolour breaking AdS minima pulls up the value of SUSY masses.

It is important to remark that the absence of consistent AdS non-SUSY vacua is at the moment only a conjecture, although in agreement with the string examples examined up to now. Furthermore, the results rely on the assumption that the obtained AdS SM vacua are stable. Although indeed the obtained minima appear stable, if the theory e.g. is 
embedded into a string landscape, one cannot rule out transitions into other lower energy vacua which might exist in the landscape. This issue is discussed in [39]. It is difficult to quantify, given our ignorance of the structure of the landscape. In the present paper we assume that those transitions are absent and the AdS minima are stable, and explore what the physical consequences would be.

The structure of this paper is as follows. In the next section we review the case of the compactification of the SM on a circle and the origin of the corresponding bounds on neutrino masses and the cosmological constant, exploring also values of the radii up to the EW scale. We then consider the structure of the potentials at small $R$ with fixed Wilson lines, as would appear in a compactification on the segment $S^{1} / Z_{2}$. In section 3 we discuss the compactification of the SM on $T^{2} / Z_{4}$ and show how the SM has vacua of Type $D$ and hence would be incompatible with quantum gravity. In section 4 we show how such vacua are not present in the SUSY case and how one recovers the neutrino mass bounds if $\mathrm{U}(1)_{B-L}$ (or a discrete subgroup) is gauged at some level. New vacua may also lead to constraints on the SUSY spectrum. We leave section 5 for our conclusions and outlook. In appendix A we discuss the computation of the Casimir potential in the SM on the circle, including also the EW degrees of freedom. In appendix B we work out some results for the compactification on the $T^{2} / Z_{4}$ orbifold. Appendix $\mathrm{C}$ contains a discussion on the consistency of assuming a flat non-compact background in the computation of Casimir energies. Finally, appendix D contains a table with the MSSM spectrum used to draw some of the plots.

\section{The $\mathrm{SM}$ in $3 \mathrm{D}$}

In this section we first study the compactification of the SM coupled to Einstein gravity with a cosmological constant down to 3D on the circle. We begin by exploring the regions with the compact radius larger than the electron wave length and then regions with smaller radius, going up to the EW scale. The resulting theory has one radius, one Higgs and four Wilson line scalar variables. We reproduce the local minima associated to the neutrino region and study the potential at shorter radii. The potential features runaway directions in the presence of Wilson line moduli that may lead to decay of the neutrino vacua through tunneling. We also discuss the structure of the radius effective potential in the case with frozen Wilson lines. This would happen in particular in theories compactified in the segment $S^{1} / Z_{2}$ and serves as an appetizer to the case of compactifications on $T^{2} / Z_{N}$ which are discussed in the next section. In this case it is easier the search for minima for the radius potential, since then all Wilson lines are fixed.

\subsection{The SM on the circle I $\left(R \gtrsim 1 / m_{e}\right)$}

We first briefly summarize and extend some of the results in $[39,41]$ so that the reader better understands the further results presented in this paper. In [41] the compactification of the SM Lagrangian to lower dimensions was considered. One of the purposes of that work was to show how the notion of landscape of vacua should not be associated exclusively to string theory, but even the SM itself has a wealth of vacuum solutions when compactified to $3 D$ or 
$2 D$. They concentrated in the deep infrared sector of the SM, below the electron threshold, in which the only relevant particles are the photon, the graviton and the neutrinos. They found that, depending on the value of the neutrino masses, the SM possesses local minima both on $3 \mathrm{D}$ and $2 \mathrm{D}$, with very large compactification radii of order $R \simeq 1 / m_{\nu}$. The appearance of these minima goes as follows. The action of the pure gravity action reduced to $3 \mathrm{D}$ in a circle is given by

$$
S_{G R}=\int d^{3} x \sqrt{-g_{3}}(2 \pi)\left[\frac{1}{2} M_{p}^{2} \mathcal{R}_{(3)}-\frac{1}{4} R^{4} W_{\mu \nu} W^{\mu \nu}-M_{p}^{2}\left(\frac{\partial R}{R}\right)^{2}-\frac{\Lambda_{4}}{R^{2}}\right] .
$$

Here $M_{p}$ is the $4 \mathrm{D}$ reduced Planck mass, $M_{p}=\left(8 \pi G_{N}\right)^{-1 / 2}$ and $\Lambda_{4}$ is the $4 \mathrm{D}$ cosmological constant. The action of the graviphoton of field strength $W_{\mu \nu}$ is also shown. There is a runaway potential inherited from the $4 \mathrm{D}$ cosmological term. Nevertheless, the cosmological constant is so small that the quantum contribution of the lightest SM modes to the effective potential becomes relevant. Their contribution at one-loop level can be identified with the Casimir energy, which for a non-interacting massless field with periodic boundary conditions is found to be:

$$
V_{p}(R)=\mp \frac{n_{p}}{720 \pi} \frac{1}{R^{6}}
$$

where $n_{p}$ counts the number of degrees of freedom of the particle. For bosons the negative sign applies, whereas for fermions the sign is positive. The only massless states in the $\mathrm{SM}$ are the photon and the graviton, so in the absence of any other light particle it is clear that the potential becomes unstable and negative for small $R$. However, if there are fermions with a mass of order $m \gtrsim \Lambda_{4}^{1 / 4}$, minima can develop. The Casimir potential for a non-interacting massive particle of spin $s_{p}$ and mass $m_{p}$ has the form [41]

$$
V_{p}(R)=(-1)^{2 s_{p}+1} n_{p} \frac{m_{p}^{2}}{8 \pi^{4} R^{4}} \sum_{n=1}^{\infty} \frac{K_{2}\left(2 \pi n R m_{p}\right)}{n^{2}} .
$$

Here $K_{2}(x)$ is a modified Bessel function of the second kind. This gives us the leading quantum contribution to the radion potential. As long as couplings remain perturbative, higher loop corrections will be small and unimportant. This excludes the region close to the QCD scale in which non-perturbative techniques would be appropriate. We will thus concentrate in the potential above or below the QCD scale. In the latter case we will count pions and kaons as elementary.

The Bessel function decays exponentially for large argument. For this reason, to work out the structure of the potential around the scale $\Lambda_{4}^{1 / 4}$ within the SM, only the neutrinos will be relevant, since heavier particles like the electron contribute in a negligible way to the local potential. Neutrino oscillation experiments tell us what are the mass differences between neutrino masses, but not their Dirac or Majorana character. We do not know whether the hierarchy of the masses is normal (NH) or inverted (IH). Experimental constraints tell us for the neutrino mass differences that [43]

$$
\begin{aligned}
& \Delta m_{21}^{2}=(7.53 \pm 0.18) \times 10^{-5} \mathrm{eV}^{2}, \\
& \Delta m_{32}^{2}=(2.44 \pm 0.06) \times 10^{-3} \mathrm{eV}^{2}(\mathrm{NH}), \\
& \Delta m_{32}^{2}=(2.51 \pm 0.06) \times 10^{-3} \mathrm{eV}^{2}(\mathrm{IH}) .
\end{aligned}
$$




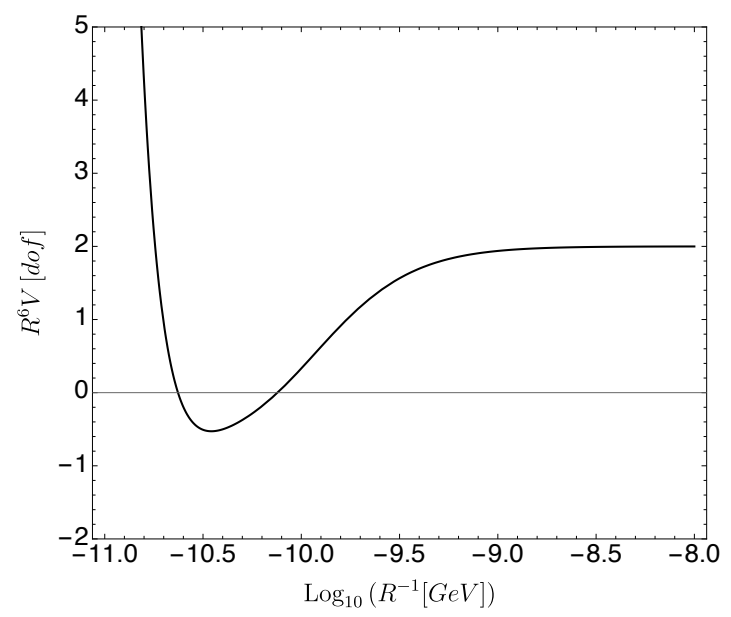

(a)

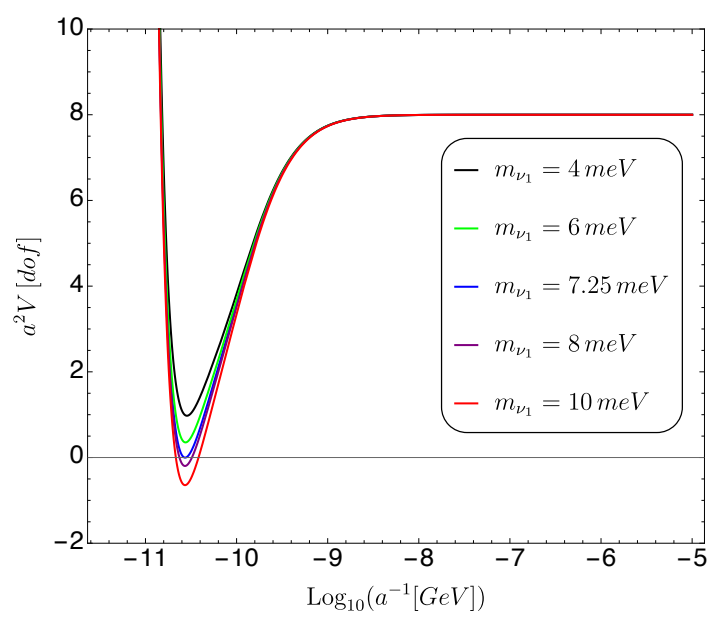

(b)

Figure 1. (a) Radion effective potential for Majorana neutrinos. The lightest neutrino is considered massless and a minimum develops. For higher energies, the neutrinos behave as massless particles. We have 4 bosonic and 6 fermionic degrees of freedom so, in the massless limit, $R^{6} V$ (in units of degrees of freedom) tends to 2. (b) Radion effective potential for Dirac neutrinos. The different lines correspond to several values for the lightest neutrino mass $m_{\nu_{1}}$. We have 4 bosonic and 12 fermionic degrees of freedom so, in the massless limit, $R^{6} V$ tends to 8 . It is important to remember that positive valued minima in this plot do not necessarily correspond to dS minima of $V$. However, negative minima do correspond to AdS minima of $V$.

We do not know what the mass of the lightest neutrino is, and it is not experimentally excluded that it could be massless. We use the above neutrino constraints in computing the radion potential and plotting the figures. We always plot the potential divided by the contribution of a single massless degree of freedom, so that we can interpret it as the number of effective degrees of freedom. Thus, the potential in our plots appears multiplied by $R^{6}$ times a certain constant. Care must be taken when extracting physical conclusions from these plots, since minima of $R^{6} V$ need not be minima of $V$. However, an AdS minima of $R^{6} V$ which eventually turns positive (in all directions), always corresponds to an AdS minima of $V$.

If neutrinos are Majorana and are given periodic boundary conditions, we have six fermionic degrees of freedom which at small $R$ dominate over the photon/graviton four degrees of freedom, so that the potential grows. However when going to values $R \simeq 1 / m_{\nu_{1}}$ an AdS minimum is always created, irrespective of the neutrino masses, since only two neutrino degrees of freedom can become sufficiently light and those cannot overwhelm the bosonic contribution at large $\mathrm{R}$, see figure $1(\mathrm{a})$. On the other hand if neutrinos are Dirac, up to 4 neutrino degrees of freedom may be sufficiently light to compensate for the four bosonic ones. In this case minima forms or not depending on the value of the lightest neutrino mass, see figure $1(\mathrm{~b})$.

If we apply the principle of no AdS non-SUSY vacua we reach the conclusion that neutrino Majorana masses are not possible and we obtain bounds for the lightest neutrino 
Dirac mass $m_{\nu_{1}}>7.7 \times 10^{-3} \mathrm{eV}$ for $(\mathrm{NI})$ and $m_{\nu_{3}}>2.56 \times 10^{-3} \mathrm{eV}$ for $(\mathrm{IH})$, see [39] for details. ${ }^{2}$

As we mentioned before, another interesting implication is that within this scheme there is a lower bound on the value of the $4 \mathrm{D}$ cosmological constant [39], qualitatively $\Lambda_{4} \gtrsim m_{\nu_{1}}^{4}$. This is interesting because it is the first argument implying a non-vanishing $\Lambda_{4}$ only on the basis of particle physics, with no cosmological input.

A further implication concerns the fine-tuning issue of the Electro-Weak (EW) scale in the SM. Indeed, the mentioned upper bounds on neutrino masses imply an upper bound on the Higgs vev, for fixed Yukawa coupling. Thus SM versions with a Higgs sector leading to Higgs vevs above $1 \mathrm{TeV}$ would be in the swampland, since larger vevs implies larger neutrino masses and hence AdS vacua would form in 3D or 2D [39]. In particular for Dirac neutrino masses one has a bound

$$
|\langle H\rangle| \lesssim 1.6(0.4) \frac{\Lambda_{4}^{1 / 4}}{Y_{\nu_{1}}},
$$

for $\mathrm{NH}(\mathrm{IH})$ neutrinos. Here $Y_{\nu_{1}}$ is the lightest neutrino Yukawa coupling and $\Lambda_{4}$ the $4 \mathrm{D}$ cosmological constant. With values of $\Lambda_{4}$ as observed in cosmology, larger values for the EW scale would necessarily lead, for fixed Yukawa, to undesired AdS minima. From the low-energy Wilsonian point of view the smallness of the EW scale looks like an enormous fine-tuning, but this would be a mirage since field theory parameters leading to larger EW scale would not count as possible consistent theories. The smallness of the EW scale compared to the Planck scale is here tied up to the smallness of the cosmological constant. Note that here we are taking the value of $\Lambda_{4}$ fixed to its observed value while varying $H$. This may be motivated by the fact that anthropic arguments from galaxy formation require $\Lambda_{4}^{1 / 4} \simeq 2.25 \times 10^{-3} \mathrm{eV}$ within a factor of order 4 [44, 45]. So we vary $H$ while keeping the cosmological constant around its measured present value.

Having Dirac neutrinos rather than Majorana departs from the most common scenario to understand the smallness of neutrino masses, the seesaw mechanism. In the present approach the lightest neutrino is very light to avoid that an AdS vacuum forms. Its mass is bounded from above by $m_{\nu_{1}} \lesssim \Lambda_{4}^{1 / 4}$, giving an explanation for the apparent numerical coincidence of neutrino and cosmological constant scales. Strictly speaking, it is only necessary for one of the three neutrinos to be that light. However, if neutrino Yukawa couplings of the different generations are correlated, as they are for the charged leptons and quarks, this condition will drag all of the neutrinos to be very light, as observed. ${ }^{3}$ Still, one would like to have in addition an explanation to why the neutrino Yukawa couplings are

\footnotetext{
${ }^{2}$ Note that what counts is that the number of degrees of freedom of the lightest neutrino should be 4 . Thus it may also be pseudo-Dirac, i.e. it may have both Dirac and Majorana mass contributions of the same order of magnitude. In this case $\nu$-less double $\beta$-decay could be posible.

${ }^{3}$ Note in this respect that obtaining Majorana neutrino masses in string compactifications has proven to be a notoriously difficult task both in heterotic and Type II orientifold constructions. In the latter, Majorana neutrino masses may in principle appear from string instanton effects [46, 47]. However, a dedicated search [48] for such instanton effects in a large class of MSSM-like Gepner orientifolds was unsuccesful. On the other hand $\nu_{R}$ Majorana masses may appear from non-renormalizable couplings of right-handed neutrinos to scalars breaking the $B-L$ symmetry in extended gauge symmetry models.
} 
so small. As emphasized in [39], neutrino Majorana masses are still allowed if we go beyond the SM and assume there is an additional singlet Weyl fermion $\chi$ with mass $m_{\chi} \lesssim 10^{-3} \mathrm{eV}$. Then the AdS vacua is again avoided if the lightest (Majorana) neutrino is light enough. In this case the bound on the EW symmetry scale has the form [39, 40]

$$
\frac{\left(Y_{\nu_{1}}\langle H\rangle\right)^{2}}{M} \lesssim 2 \times \Lambda_{4}^{1 / 4} \longrightarrow\langle H\rangle \lesssim \frac{\sqrt{2}}{Y_{\nu_{1}}} \sqrt{M \Lambda_{4}^{1 / 4}}
$$

where $M$ is the scale of lepton number violation in the see-saw mechanism. In this case the EW scale is bounded from above by the geometric mean of the cosmological constant scale and the lepton number violation scale $M$. Thus, e.g. for $Y_{\nu_{1}} \simeq 10^{-3}$ and $M \simeq$ $10^{10}-10^{14} \mathrm{GeV}$, one gets $\langle H\rangle \lesssim 10^{2}-10^{4} \mathrm{GeV}$. This possibility is interesting because, since it uses the see-saw mechanism, no hierarchically small Yukawas are needed. In the rest of this paper it will not be relevant whether neutrinos are Dirac or Majorana $+\chi$, but we think it is interesting to keep in mind the different possibilities.

All these constraints obtained imposing the absence of AdS local minima assume that these vacua are absolutely stable. A possible source of instabilities may in principle arise if there are lower minima or runaway directions at smaller radion values (higher thresholds) contributing to the Casimir potential [42]. This is an important motivation to go to the orbifold case, as we will discuss in section 3 .

\subsection{The SM on the circle II $\left(1 / m_{e} \gtrsim R \gtrsim 1 / M_{\mathrm{EW}}\right)$}

In this section we study the one-loop effective potential of the full Standard Model with massive Dirac neutrinos and minimal coupling to gravity compactified in a circle. Our calculations were carried out using the Background Field Method, in the spirit of [49]. Some details on the computations can be found in appendix A. We parameterize the metric as in the usual Kaluza-Klein ansatz:

$$
g_{\mu \nu}=\frac{1}{R^{2}}\left[\begin{array}{cc}
g_{i j}+R^{4} B_{i} B_{j} & R^{4} B_{i} \\
R^{4} B_{j} & R^{4}
\end{array}\right] .
$$

We will set the graviphoton $B_{i}=0$ from the start, since its vev is zero and it does not contribute to the effective potential. The scalar fields in the 3D Effective Action are the radion $R$, the Higgs field $H$ (whose vev is $\mathrm{v} \simeq 246 \mathrm{GeV}$ ) and the Wilson lines of all the gauge bosons in the Cartan Subalgebra of $\mathrm{SU}(3)_{C} \times \mathrm{SU}(2)_{L} \times \mathrm{U}(1)_{Y}$ : two gluon Wilson lines $\left(G_{1}, G_{2}\right)$, the photon $A$ and the $Z$ boson. To perform the compactification we need to expand our fields $\phi$ in a basis that respects the boundary condition:

$$
\phi\left(x_{i}, y+2 \pi\right)=e^{i 2 \pi\left(\frac{1-z}{2}\right)} \phi\left(x_{i}, y\right),
$$

since $y \in(0,2 \pi)$. Fermions can have either periodic $z=1$ or antiperiodic $z=0$ boundary conditions, while bosons are only allowed to have periodic ones. We use Fourier harmonics:

$$
\phi\left(x_{i}, y\right)=\sum_{n=-\infty}^{\infty} \phi_{n}\left(x_{i}\right) e^{i 2 \pi\left(n+\frac{1-z}{2}\right) y} .
$$


The relevant classical contribution to the effective potential in three dimensions consists of a term coming from the $4 \mathrm{D}$ cosmological constant, the Higgs potential and a mass term for the $Z^{0}$ boson Wilson line $(Z)$. We will neglect the effects of a non-vanishing 3D curvature in the Casimir computation. We argue in appendix $\mathrm{C}$ that those effects may be safely neglected as long as the radii are not exceedingly small. The one-loop potential can be consistently identified with the Casimir energy $V_{p}$ of the different particles. One then has

$$
\begin{aligned}
V\left[R, H, Z, A, G_{1}, G_{2}\right]=\frac{1}{R^{2}}[ & 2 \pi \Lambda_{4}+\frac{1}{2} m_{H}^{2}(H-\mathrm{v})^{2}+\lambda \mathrm{v}(H-\mathrm{v})^{3}+\frac{\lambda}{4}(H-\mathrm{v})^{4} \\
& \left.+\frac{1}{2} M_{Z}^{2} \frac{H^{2}}{\mathrm{v}^{2}} Z^{2}\right]+\sum_{p} V_{p} .
\end{aligned}
$$

As explained in appendix A, the contribution of each particle to the Casimir potential can always be written as:

$$
V_{p}=(-1)^{2 s_{p}+1} n_{p} \frac{-i}{2} \sum_{n=-\infty}^{\infty} \int \frac{d^{3} p}{(2 \pi)^{3}} \log \left(-p^{2} R^{2}+\left(m_{p} \frac{H}{\mathrm{v}}\right)^{2}+(n+\theta)^{2} R^{2}\right) \text {, }
$$

where $s_{p}, n_{p}, m_{p}$ are the spin, number of degrees of freedom and mass of the particle and $\theta$ is a function of the Wilson lines and $z_{p}$. Note that the masses of the particles appear multiplied by the Higgs divided by its vev, $v$. The only exception is the Higgs field, whose mass is changed by the non-linear couplings to the $Z^{0}$ Wilson line and to itself. Using the same techniques as e.g. $[42,50]$ we regularize the integration and the sum using the analytic continuation of generalized $\zeta$-functions. After regularization, the contribution to the Casimir energy for each particle reads:

$$
V_{p}=(-1)^{2 s_{p}+1} n_{p} \frac{m_{p}^{2}}{8 \pi^{4} R^{4}} \sum_{n=1}^{\infty} \frac{K_{2}\left(2 \pi n R m_{p}\right)}{n^{2}} \cos (2 \pi n \theta) \equiv(-1)^{2 s_{p}+1} n_{p} V_{\mathcal{C}}\left[R, m_{p}, \theta\right]
$$

$K_{2}(x)$ decays exponentially for large $x$, which means that at low energies, the contribution of very massive particles to the Casimir energy is highly suppressed. For massless particles with $\theta=0$ we have

$$
V_{\mathcal{C}}[R, 0,0]=\frac{1}{720 \pi R^{6}}
$$

while for $\theta=\frac{1}{2}$ we have

$$
V_{\mathcal{C}}\left[R, 0, \frac{1}{2}\right]=-\frac{7}{8} \frac{1}{720 \pi R^{6}}
$$

We cannot use this one-loop formula to study the minima of the potential around $\sim 1 \mathrm{GeV}$, since perturbation theory breaks down for the strong interaction. Below the QCD scale, we consider an effective field theory of pions and kaons. For completeness, we give here 
the formula above the QCD scale:

$$
\begin{aligned}
V= & -\frac{(2+2)}{720 \pi R^{6}}-\frac{2}{8 \pi^{5} R^{6}} \sum_{n=1}^{\infty} \frac{1}{n^{4}}\left\{2+2 \cos \left(2 \pi n G_{1}\right)\right. \\
& +2 \cos \left(2 \pi n\left(\frac{1}{2} G_{1}+\frac{3}{2} G_{2}\right)\right)+2 \cos \left(2 \pi n\left(\frac{1}{2} G_{1}-\frac{3}{2} G_{2}\right)\right) \\
& +4 \sum_{\text {lept }} V_{\mathcal{C}}\left[R, m_{l}\left(\frac{H}{\mathrm{v}}\right), \frac{1-z_{l}}{2}+Q_{l} A+\frac{1}{2}\left(g_{L}^{l}+g_{R}^{l}\right) Z\right] \\
+ & 4 \sum_{\text {flav }}\left\{V_{\mathcal{C}}\left[R, m_{f}\left(\frac{H}{\mathrm{v}}\right), \frac{1-z_{f}}{2}+Q_{f} A+\frac{1}{2}\left(g_{L}^{f}+g_{R}^{f}\right) Z+\frac{1}{2}\left(G_{1}+G_{2}\right)\right]\right. \\
& +V_{\mathcal{C}}\left[R, m_{f}\left(\frac{H}{\mathrm{v}}\right), \frac{1-z_{f}}{2}+Q_{f} A+\frac{1}{2}\left(g_{L}^{f}+g_{R}^{f}\right) Z-\frac{1}{2}\left(G_{1}-G_{2}\right)\right] \\
& \left.+V_{\mathcal{C}}\left[R, m_{f}\left(\frac{H}{\mathrm{v}}\right), \frac{1-z_{f}}{2}+Q_{f} A+\frac{1}{2}\left(g_{L}^{f}+g_{R}^{f}\right) Z-G_{2}\right]\right\} \\
& -6 V_{\mathcal{C}}\left[R, M_{W} \frac{H}{\mathrm{v}}, A-c_{w}^{2} Z\right]-3 V_{\mathcal{C}}\left[R, M_{Z} \frac{H}{\mathrm{v}}, 0\right] \\
& -V_{\mathcal{C}}\left[R, m_{H}\left(\frac{-1}{2}+\frac{3}{2}\left(\frac{H}{\mathrm{v}}\right)^{2}+\frac{m_{Z}^{2}}{2 m_{H}^{2}} Z^{2}\right)^{1 / 2}, 0\right] .
\end{aligned}
$$

The first two lines include the contribution of the graviton, photon and the 8 gluons. The third line include the one of leptons and the following three lines the 3 colours of quarks. The seventh line gives the contribution of massive $W^{ \pm}$'s and the $Z^{0}$. The last line is the contribution of the Higgs. Here $g_{L, R}$ give the left and right $Z^{0}$ couplings to fermions, $c_{w}=\cos \theta_{W}$ and $Z, A$ are the $Z^{0}$ and photon Wilson lines. This formula is the generalization of the one in appendix B1 in [41], since it includes the $Z^{0}$ Wilson line and the Higgs field. Notice that our gluon Wilson lines are expressed in terms of the basis chosen in [41] as

$$
\begin{aligned}
& G_{1}=G_{\phi}^{1}-G_{\phi}^{2}, \\
& G_{2}=G_{\phi}^{1}+G_{\phi}^{2} .
\end{aligned}
$$

eq. (2.17) in principle allows for a detailed study of the SM action on the circle. In practice, it is a complicated function of six scalar fields and the fermions boundary conditions, and a full analysis is a formidable task. However a number of conclusions may already be drawn without fully analyzing the complete potential. In figure 2 we plot the value of $R^{6} V$ in the Higgs-Radion plane with Wilson lines fixed to zero and periodic boundary conditions for fermions. As expected the minima stays always at $H=\mathrm{v}$. Moreover, this conclusion also holds for different values of the Wilson lines. Thus, we will set the Higgs equal to its tree level vev from now on.

Even tough the potential in terms of the four Wilson lines is still complicated, we can see with a simple example that the neutrino vacua may become unstable to tunneling due to the existence of runaway directions at smaller radius $\mathrm{R}$. In figure 3 we show the effective potential as a function of the photon Wilson line and the Radion, with the Higgs field at the minima and all other Wilson lines set to zero. The point illustrated by this plot is that the effect of the Wilson lines on the Casimir potential of a particle is to gradually change 


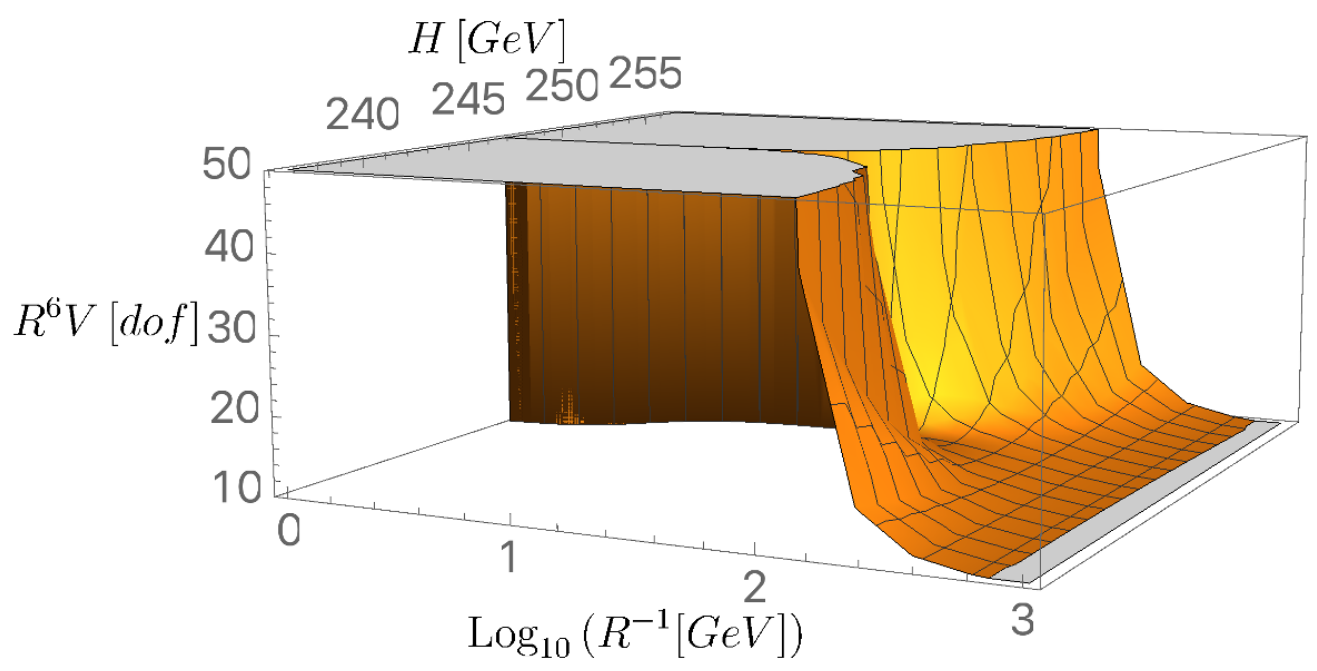

Figure 2. Effective potential with the Wilson lines fixed to zero, as a function of the Radion and the Higgs. The tree level potential dominates and the Higgs is not displaced from its tree level minimum by the one-loop corrections. This behavior is independent of the particular value of the Wilson lines Although not very visible in the plot, the Higgs minimum remains at the same location as $R^{-1}$ increases.

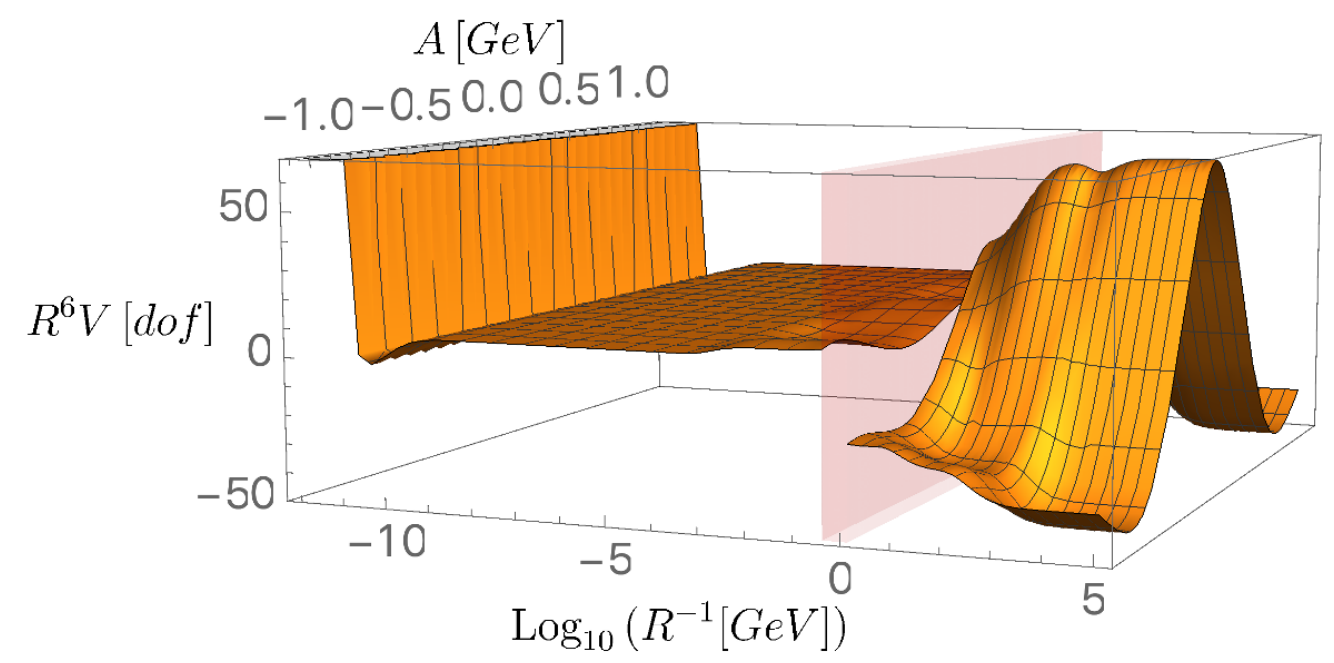

Figure 3. Effective potential as a function of the photon Wilson line and the Radion, with the Higgs field at the minima and all other Wilson lines set to zero. Notice that a runaway develops along the Radion direction for a non-zero value of the Wilson line. 
its sign. More precisely, it transforms periodic particles into antiperiodic ones (there is also a factor $\frac{7}{8}$ involved). The results of [39] correspond to the $A=0, R^{-1}<m_{e}$ part of the plot, where we still see the neutrino minima. Moving on to higher energies along $A=0$, we see a small bump corresponding to the four degrees of freedom of the electron. After going through the QCD transition, where we do not know the shape of the potential, we observe a fast increase caused by the copiously unleashed quark degrees of freedom. At around $100 \mathrm{GeV}$, the $W, Z^{0}$ and the Higgs slow down a bit the potential and, finally, the top quark increases it a bit more. Unless new physics is introduced, $R^{6} V$ remains constant for higher energies, so $V$ keeps increasing, at least while our one-loop approximation remains valid. On the other hand, if we displace the Wilson line $A$ from 0 , these conclusions are changed. In particular, for $A=\frac{1}{2}$ or $\frac{3}{4}$, the sign of each contribution is reversed. As we can see in figure 3 this leads inevitably to a negative potential. After the top quark scale, $R^{6} V$ remains constant for higher energies, so $V$ keeps decreasing. This runaway behaviour of the potential could mean that the neutrino minima is actually metastable, as pointed out in [42]. In fact, to be sure that this vacuum is unstable we should be able to find the bounce interpolating from the vacuum to the runaway direction, which is a complicated question in such a complicated potential. We will however conservatively assume that indeed the vacua are metastable. This would be consistent with the OV conjecture and would lead to no constraints on neutrino masses nor on the hierarchy.

If we want to search for constraints on observable physics using the OV conjecture we would need Type P SM vacua in which this potential tunneling in the Wilson line directions is absent. That would be the case of vacua in which the Wilson lines are frozen or projected out in such way that these decay directions disappear. In the rest of this paper we will focus on this class of models. For reasons soon to be explained, a good option is the compactification on the orbifold $T^{2} / Z_{4}$. But before going to that case we explain in the next section the case of $3 \mathrm{D}$ vacua with Wilson line moduli fixed, which will give us intuition for the more elaborate $T^{2} / Z_{4}$ case.

\subsection{Fixing the Wilson lines. The segment $S^{1} / Z_{2}$}

In this section we describe the structure of the radius scalar potential at shorter distances, up to the EW scale, setting the Wilson line moduli to zero by hand, looking for new features beyond the neutrino local minima. In figure 4 (a) we show the product $R^{6} V$ for the Standard Model with all fermions having periodic boundary conditions, using the formulae introduced in the previous section for the circle. We see that the only minimum we may obtain is the one associated with the bounds in the neutrino masses (in the Majorana case). Above the neutrino scale the potential grows smoothly until it reaches the QCD region, around one $\mathrm{GeV}$, where non-perturbative physics become important and the oneloop Casimir computation is not a good description (this is denoted by the red, vertical band in the plots). Above the QCD scale perturbation theory again makes sense and the potential keeps growing indefinitely. The small well in the upper part of the figure corresponds to the $W^{ \pm}, Z^{0}, H^{0}$ threshold, while the final increase corresponds to the top quark threshold. Consequently, the neutrino minima seems stable and the bounds on neutrino masses would apply. 
Let us consider now the case in which some of the fermions are instead assigned antiperiodic boundary conditions. In principle one could naively say that we can assign arbitrary boundary conditions to each fermion multiplet of the SM and each generation. However, due to the presence of Yukawa couplings and generation mixing all quarks must have the same boundary conditions, and likewise for the leptons. So there are four options according to the (quark, lepton) boundary conditions: $(P, P),(A P, P),(P, A P)$ and $(A P, A P)$. The last possibility, with all fermions antiperiodic does not produce any locally stable vacuum since the potential always decreases and even if they appeared they seem unstable to decay into a bubble of nothing [51]. It is easy to see that the $(A P, P)$ case will lead to a runaway potential. In the SM there are more degrees of freedom associated with quarks than leptons and, since for small radius the antiperiodic fermion contribution picks up a factor $-\frac{7}{8}$, the potential will grow large and negative. The remaining case with antiperiodic leptons and periodic quarks is illustrated in figure 4(b). As $R$ decreases the potential decreases steadily until the QCD transition. Once passed the hadron region, the potential grows up monotonously due the appearance of all quark degrees of freedom, which dominate the potential as $R \rightarrow 0$. Although the proximity of the QCD deconfining region does not allow for a computation of the precise location of the minimum, one certainly expects a minimum to develop. No lower minima develops for other regions of $R$ and hence that minimum seems stable. This gives a Type $D$ minimum since it cannot be avoided by fixing any free parameter of the Standard Model. For example, the masses of all the SM particles around the QCD scale are known, so there is no way to eliminate it by varying masses, as it happens in the neutrino case. As it is, the SM would be inconsistent with the OV conjecture and would not be embeddable into a consistent theory of quantum gravity. Still, up to here we have not provided a reason why the Wilson lines should be fixed. Those are fixed in the class of $T^{2} / Z_{N}$ vacua considered in the next section, and again this class of AdS vacua will appear. Moreover, we will see in section 3 that there are even more AdS vacua which will confirm the difficulties in embedding the SM into quantum gravity.

A way to avoid the AdS vacua above would be to go beyond the minimal SM and add e.g. additional massive bosons above the EW scale so that the potential develops a runaway behavior as $R \rightarrow 0$, making the dangerous minima unstable. Indeed, consider the limit $m R \rightarrow 0$ of the Casimir potential with no Wilson lines. To first order in the masses of the particles one obtains

$$
\begin{aligned}
V= & -\frac{(2+2+2 \times 8)}{720 \pi R^{6}}+\sum_{P}(-1)^{2 s_{p}+1} \frac{n_{P}}{8 \pi} \times\left\{\frac{1}{90 R^{6}}-\frac{m_{p}^{2}}{6 R^{4}}\right\} \\
& +\sum_{A P}(-1)^{2 s_{p}+1} n_{P} \times\left\{-\frac{7}{8} \frac{1}{90 R^{6}}+\frac{1}{2} \frac{m_{p}^{2}}{6 R^{4}}\right\}
\end{aligned}
$$

where we denote by $P$ the particles with periodic boundary conditions and by AP the fermions with antiperiodic boundary conditions. The first term corresponds to the massless graviton, photon and gluons. It is clear that if we add a sufficiently large number of periodic bosons the potential will become negative and unbounded from below as $R \rightarrow 0$, making the potential unstable, and hence leading to no violation of the OV conjecture. This scenario is not particularly well motivated. 


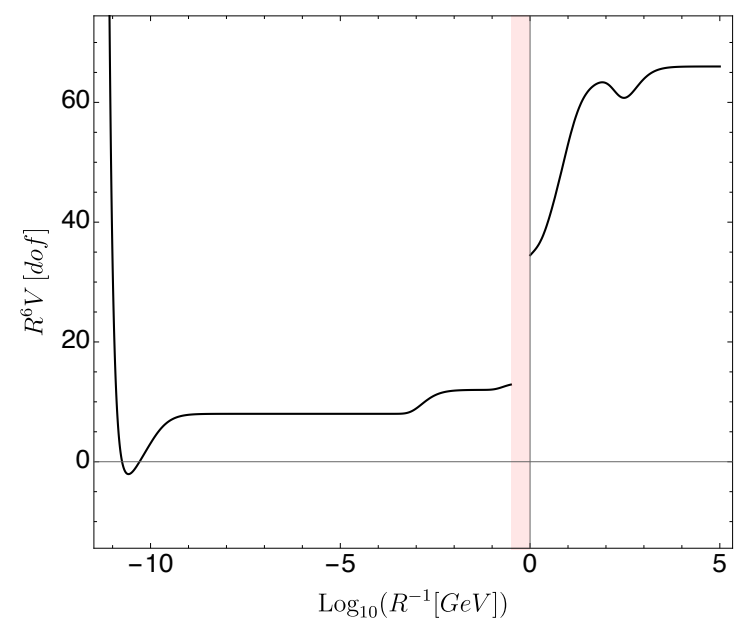

(a)

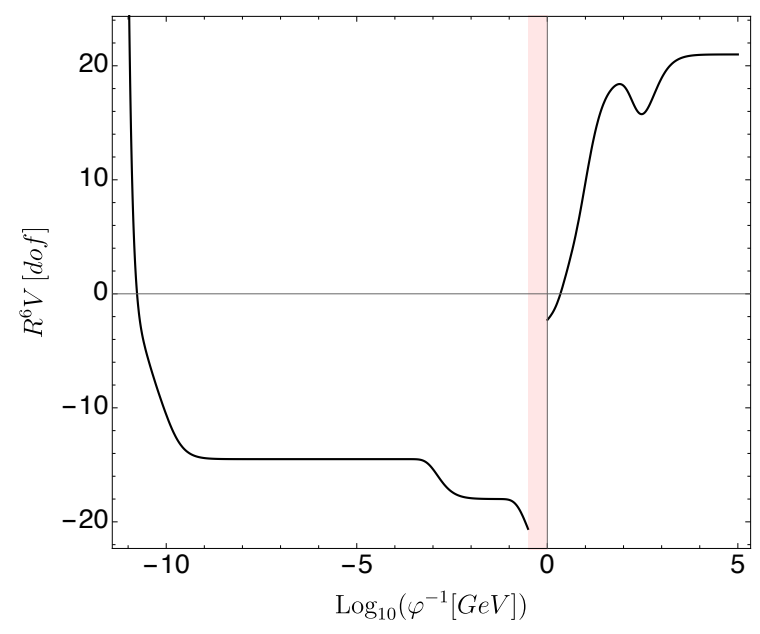

(b)

Figure 4. (a) Effective potential of the Standard Model compactified in a circle with periodic boundary conditions for all particles, the Higgs fixed at its minimum and all Wilson lines turned off. There is no runaway solution. The only AdS minimum we have is the one that would be associated with neutrino bounds. (b) Effective potential of the Standard Model with periodic boundary conditions for all particles except for the leptons. The Higgs is fixed at its minimum and all Wilson lines are turned off. There is no runaway solution. We find an AdS minimum which cannot be avoided by constraining any free parameters of the Standard Model.

We will argue in section 4 that a more elegant option is to embed the SM into a SUSY version like the MSSM. It turns out that in the SUSY case this vacuum is not possible since quarks and leptons are forced to have the same boundary conditions. But we will also see that SUSY also avoids new classes of AdS vacua which exist in the orbifold case. Considering SUSY is an attractive possibility because of another important reason. It is well known that the Higgs potential of the SM may have a second high energy minimum at scales above $10^{10} \mathrm{GeV}[52-57]$. This $4 \mathrm{D}$ minimum is would be in AdS and, if stable, would be again inconsistent with the AdS-phobia condition. Although it would be easy to save the SM by the addition of some BSM physics like e.g. additional singlet scalars eliminating the high energy vacuum, SUSY seems to be a more attractive option. If the SUSY breaking scale is below $10^{10} \mathrm{GeV}$ the high energy Higgs minimum does not develop (see e.g. $[58,59]$ ), since the SUSY potential is positive definite. We will come back to the SUSY case in section 4 .

Up to now we have just set the Wilson lines to zero by hand and have analyzed the resulting potential as a function of $R$ and the Higgs field. We discuss in what follows how the Wilson lines could be fixed. The simplest option seems to be to compactify on the segment $S^{1} / Z_{2}$, where $Z_{2}$ is a reflection with respect to one spatial dimension $y$. We have boundary conditions under $y \rightarrow-y$ for scalar, vector and spinor fields:

$$
\begin{aligned}
\phi\left(x_{i}, y\right) & =\phi\left(x_{i},-y\right) \\
A_{\mu}\left(x_{i}, y\right) & =\left\{A_{i}\left(x_{i},-y\right),-A_{3}\left(x_{i},-y\right)\right\} \\
\Psi\left(x_{i}, y\right) & = \pm \gamma^{3} \gamma^{5} \Psi\left(x_{i},-y\right) .
\end{aligned}
$$


We see that the Wilson lines are projected out, they are forced to be zero. Furthermore, half of the Fourier modes are projected out, and one can check that the Casimir potential is reduced by a factor 2 . We can use directly the formulas for the circle if we take into account these two points since nothing else changes. In this orbifold the contribution to the Casimir potential of each particle $V_{\mathcal{C}}\left[R, m_{p}, \theta\right]$ is thus very simple. The results for the scalar potential would be qualitatively identical to the case of the circle with Wilson lines fixed to zero.

We could envisage to compactify the SM on the segment and obtain the results above with fixed Wilson lines. However there is a technical obstruction in this simple situation. Indeed, reflection with respect to a single coordinate (or in general, parity) is not a symmetry of the SM (since it reverses chirality) and we cannot twist the theory with respect to it, unless we do some modification or extension. Such an extension could be the embedding of the SM in a theory which is left-right symmetric like $\mathrm{SU}(3) \times \mathrm{SU}(2)_{L} \times \mathrm{SU}(2)_{R} \times \mathrm{U}(1)$, $\mathrm{SU}(4) \times \mathrm{SU}(2)_{L} \times \mathrm{SU}(2)_{R}$ or $\mathrm{SO}(10)$. But then we have to include the Higgsing down to the SM in the computations. We will prefer instead to go to $2 \mathrm{D}$ orbifold compactifications $T^{2} / Z_{N}$, with $Z_{N}$ a discrete rotation in two dimensions, which is always a symmetry of the $4 \mathrm{D}$ SM. We will first discuss the case of the torus, from which it is easy to obtain the results for the orbifolds.

\section{The SM on the $T^{2} / Z_{4}$ orbifold}

In this section we consider the case of the compactification of the SM on the torus $T^{2}$ and then on the orbifold $T^{2} / Z_{4}$ which is closely related.

\subsection{The SM on the $T^{2}$ torus}

In this section we first review some of the most relevant features and results of the compactification of the SM on a torus. Compactifications of the SM on a torus have been considered in [41, 60,61] and [39, 42].

As in the circle, to obtain the potential of the moduli we will consider the scalar potential of the SM plus Einstein gravity compactified on the torus and the one-loop contribution coming from the Casimir energy of the corresponding particles.

We take for the distance element in 4 dimensions,

$$
d s^{2}=g_{\alpha \beta} d x^{\alpha} d x^{\beta}+B_{\alpha i} d x^{\alpha} d y^{i}+t_{i j} d y^{i} d y^{j},
$$

with $g_{\alpha \beta}$ the 2-dimensional metric, $B_{\alpha 2}$ and $B_{\alpha 3}$ the two graviphotons, which will be set to zero from now on for the same reason as in the circle, and $t_{i j}$ the metric on the torus, which reads

$$
t_{i j}=\frac{a^{2}}{\tau_{2}}\left[\begin{array}{cc}
1 & \tau_{1} \\
\tau_{1} & |\tau|^{2}
\end{array}\right] .
$$

Here $\tau=\tau_{1}+i \tau_{2}$ and $a^{2}$ are the complex structure and area moduli, respectively. The relevant piece of the effective action after dimensional reduction on the torus takes the form

$$
\left.S_{\mathrm{GR}+\mathrm{SM}}=\int d^{2} x \sqrt{-g_{2}}\left[\frac{1}{2} M_{p}^{2}(2 \pi a)^{2}\left(\mathcal{R}_{(2)}-\frac{1}{2 \tau_{2}}\left\{\left(\partial_{\alpha} \tau_{1}\right)^{2}+\partial_{\alpha} \tau_{2}\right)^{2}\right\}\right)-V\right],
$$


where $V$ includes the $4 \mathrm{D}$ cosmological constant, the tree-level and the Casimir contributions to the potential of the scalars in the theory. Notice that, as expected from the fact that the 4-dimensional graviton has two degrees of freedom, only the two complex structure moduli propagate in two dimensions, whereas the area moduli does not propagate any additional degree of freedom (i.e. it has no kinetic term in the 2D action). Moreover, unlike in the case of the circle, now we cannot perform a Weyl transformation to express the action in the Einstein frame, due to the conformal invariance of $2 \mathrm{D}$ gravity. This means that the function $V$ should not be interpreted as a canonical potential, and that we have to resort to the equations of motion in order to study the vacua of the lower dimensional theory. Even though $V$ is not minimized with respect to all the variables at the vacua of the theory, we will still call it potential. The conditions for $\mathrm{AdS}_{2}$ vacua are (see [41, 60] for details):

$$
\begin{aligned}
& V\left(a, \tau, \phi_{j}\right)=0, \quad \partial_{\tau_{1}, 2} V\left(a, \tau, \phi_{j}\right)=0, \quad \partial_{\phi_{j}} V\left(a, \tau, \phi_{j}\right)=0, \\
& \partial_{a} V\left(a, \tau, \phi_{j}\right)<0, \quad \partial_{\tau_{1}, \tau_{2}}^{2} V(a, \tau, \phi)>0, \quad \partial_{\phi_{j}}^{2} V\left(a, \tau, \phi_{j}\right)>0,
\end{aligned}
$$

where the $\phi_{j}$ represent all the other 2-dimensional scalars in the theory. Notice that these conditions are the usual minimization conditions for the potential with respect to all the scalars in the theory except $a$. These two atypical constraints (the ones in the first column) come from requiring constant field solutions to the equations of motion. Variation of the action with respect to $a$ yields the constraint

$$
\mathcal{R}_{(2)}=\frac{1}{\left(2 \pi M_{p}\right)^{2}} \frac{\partial_{a} V}{a},
$$

so that the curvature in $2 \mathrm{D}$ is fixed by the derivative of the potential with respect to a. Thus an AdS vacuum is obtained for a negative derivative. A possible new degree of freedom in the torus case is the addition of magnetic fluxes along some U(1) in the Cartan subalgebra of the SM. However, fluxes contribute enormously to the vacuum energy and do not lead to interesting vacua, see [42, 60].

Regarding the rest of $2 \mathrm{D}$ scalars that enter the potential, we have the Higgs, two Wilson lines (corresponding to the two cycles of the torus) for each of the neutral gauge fields in the SM, that is, two for the photon $A$, two for the $Z^{0}$ boson and two more for each of the two gluon fields belonging to the Cartan subalgebra of $\mathrm{SU}(3)_{C}$. The potential that we consider has the following structure

$$
\begin{aligned}
V\left[a, \tau, H, Z_{i}, A_{i}, G_{1 i}, G_{2 i}\right]= & (2 \pi a)^{2} \Lambda_{4}+(2 \pi a)^{2} V_{\mathrm{SM}}\left(H, Z_{1}, Z_{2}\right) \\
& +\sum_{p} V_{p}\left(a, \tau, H, Z_{i}, A_{i}, G_{1 i}, G_{2 i}\right) .
\end{aligned}
$$

As in the circle compactification, it consists of a piece that depends on the $4 \mathrm{D}$ cosmological constant $\Lambda_{4}$, a tree level piece for the Higgs and the $Z$ Wilson lines (which comes from the $4 \mathrm{D}$ Higgs potential and the $4 \mathrm{D}$ mass term of the $Z$ boson, respectively) and a contribution from the Casimir energy of the particles in the spectrum. Again, this last piece is the sum of the contributions coming from each particle and introduces the dependence on the geometric moduli, the Higgs and the Wilson lines to which it couples. In order to compute 
the Casimir energy we need to expand our $4 \mathrm{D}$ fields in a basis that respects the periodic $\left(z_{i}=1\right)$ or antiperiodic $\left(z_{i}=0\right)$ boundary conditions along the two cycles of the torus, that is

$$
\phi\left(x^{\alpha}, y^{i}\right)=\sum_{n_{1}, n_{2}=-\infty}^{\infty} \phi_{n_{1}, n_{2}}\left(x^{\alpha}\right) e^{2 \pi i\left[\left(n_{1}+\frac{1-z_{1}}{2}\right) y_{1}+\left(n_{2}+\frac{1-z_{2}}{2}\right) y_{2}\right]} .
$$

After inserting this expansion into the one-loop quantum effective action and integrating over the compact dimensions, the Casimir contribution of a particle with spin $s_{p}, n_{p}$ degrees of freedom and mass $m_{p}$ turns out to be:

$$
V_{p}=(-1)^{2 s_{p}+1} n_{p} \frac{-i}{2} \sum_{n_{1}, n_{2}=-\infty}^{\infty} \int \frac{d^{2} p}{(2 \pi)^{2}} \log \left[-p^{2}+\left(m_{p} \frac{H}{v}\right)^{2}+\frac{1}{a^{2} \tau_{2}}\left|\left(n_{2}+\theta_{2}\right)-\tau\left(n_{1}+\theta_{1}\right)\right|^{2}\right] .
$$

The $\theta_{i}$ are functions of the appropriate Wilson lines and boundary conditions $z_{i}$. Using dimensional or $\zeta$-function regularization techniques one arrives, after renormalizing the cosmological constant, at the following expression for the Casimir energy of each particle $[42,60]$ :

$$
\begin{aligned}
V_{p}= & \frac{(-1)^{2 s_{p}+1} n_{p}}{(2 \pi a)^{2}}\left[2 a^{2} \tau_{2} m^{2} \sum_{p=1}^{\infty} \frac{\cos \left(2 \pi p \theta_{1}\right)}{p^{2}} K_{2}\left(\frac{2 \pi p a m}{\sqrt{\tau_{2}}}\right)\right. \\
& \left.+\frac{1}{4 \pi \tau_{2}} \sum_{n=-\infty}^{\infty}\left(2 \pi \sqrt{\left(n+\theta_{1}\right)^{2} \tau_{2}^{2}+m^{2} a^{2} \tau_{2}}\left\{\operatorname{Li}_{2}\left(e^{\sigma_{+}}\right)+\operatorname{Li}_{2}\left(e^{\sigma_{-}}\right)\right\}+\left\{\operatorname{Li}_{3}\left(e^{\sigma_{+}}\right)+\operatorname{Li}_{3}\left(e^{\sigma_{-}}\right)\right\}\right)\right] \\
& \equiv(-1)^{2 s_{p}+1} n_{p} V_{\mathcal{C}}\left[a, \tau, m, \theta_{1}, \theta_{2}\right],
\end{aligned}
$$

where $\mathrm{Li}_{s}$ is a polylogarithm and

$$
\sigma_{ \pm}=2 \pi\left( \pm i\left\{-\left(n+\theta_{1}\right) \tau_{1}+\theta_{2}\right\}-\sqrt{\left(n+\theta_{1}\right)^{2} \tau_{2}^{2}+M a^{2} \tau_{2}}\right)
$$

There are five scalar fields that must be stabilized at low energies (the three geometric moduli and the two photon Wilson lines), and the number increases as the energy grows: four extra gluon Wilson lines once the QCD scale is surpassed and the Higgs plus the two $Z^{0}$ Wilson lines when the EW scale is reached. In the case of the circle we saw that the Wilson lines tend to create runaway directions that non-perturbatively destabilize any possible AdS vacua of the lower dimensional theory [42], hence ruining the possibility to obtain any constraint from AdS-phobia, we only obtain Type $S$ vacua. As we saw in subsection 2.3, setting the Wilson lines to zero simplifies things in such a way that constraints are expected to come up. This is our main motivation to consider an orbifold compactification of the torus, as the action of the $Z_{N}$ will project out the Wilson lines from the spectrum.

Let us explain why we choose to study, in particular, the $Z_{4}$ orbifold. First of all the complex structure is only fixed if the quotient is by $Z_{N}$ with $N \geq 3$. The quotient by $Z_{2}$ would project out the Wilson lines but would not fix the complex structure. In this case it is necessary to find the minima in the direction of the complex structure. This is easy to do 
when all particles have periodic boundary conditions. In this case the potential is invariant under $\mathrm{SL}(2 ; Z)$ modular transformations and therefore the extrema of the potential must be at the stationary points of the complex structure, that is, $\tau=1$ or $\tau=1 / 2+i \sqrt{3} / 2$. In fact, as it is concluded in [60], only the latter allows for the existence of minima. However, if there are both $\mathrm{P}$ and $\mathrm{AP}$ boundary conditions the combined minimization of complex structure and radius becomes less trivial. As shown in subsection 2.3, the appearance of antiperiodic boundary conditions is of some interest, because they may generate additional "exotic" AdS minima, that come out as a generic feature of the SM as we know it. For this reason, we prefer an orbifold in which the complex structure is fixed from the start. Finally, among the remaining orbifolds, the formulas for the $Z_{4}$ orbifold are the easiest ones to work with. Still the general results obtained in the next section are expected to apply for any $Z_{N}$ orbifold acting crystallographically on the torus.

\subsection{SM compactification on the $T^{2} / Z_{4}$ orbifold}

The most relevant difference between $T^{2}$ and $T^{2} / Z_{4}$ is that we need to introduce additional boundary conditions to prevent the Lagrangian from becoming multivalued as a result of the new identifications. Instead of using $a, \tau$ it is sometimes useful to use the torus radii $R_{1}, R_{2}$ and the angle $\theta$ between them as parameters:

$$
\tau=e^{i \beta} \frac{R_{2}}{R_{1}} \quad \tau_{2}=\frac{a^{2}}{R_{1}^{2}} \quad \tau_{1}=\frac{1}{R_{1}^{2}} \sqrt{R_{1}^{2} R_{2}^{2}-a^{4}} \quad a^{2}=R_{1} R_{2} \sin \beta
$$

Remember that, for consistency of the $Z_{4}$ identifications [62], the geometrical action of the orbifold must act crystallographically on the torus lattice, fixing the complex structure to $\tau=i$ and $R_{1}=R_{2} \equiv R$ or $a^{2}=R^{2}$. We have two symmetry transformations associated with the torus $\mathcal{T}$ and three non-trivial ones with the orbifold $\mathcal{P}$. Their action on the compact coordinates is given by:

$$
\begin{aligned}
& \mathcal{T}_{A}\left(y_{1}, y_{2}\right)=\left(y_{1}+R, y_{2}\right) \\
& \mathcal{T}_{B}\left(y_{1}, y_{2}\right)=\left(y_{1}, y_{2}+R\right) \\
& \mathcal{P}_{4}\left(y_{1}, y_{2}\right)=\left(-y_{2}, y_{1}\right) \\
& \mathcal{P}_{4}^{2}\left(y_{1}, y_{2}\right)=\left(-y_{1},-y_{2}\right) \\
& \mathcal{P}_{4}^{3}\left(y_{1}, y_{2}\right)=\left(y_{2},-y_{1}\right)
\end{aligned}
$$

Under any of these symmetries the scalar fields transform as $\phi(x, y) \longrightarrow \phi\left(x, y^{\prime}\right)$. It is enough to impose boundary conditions for the two translations and for the smallest rotation, of angle $\frac{\pi}{2}$. The boundary conditions for the translations imply that we can expand the modes in the usual Fourier series:

$$
\begin{aligned}
\phi\left(x_{i}, y_{1}, y_{2}\right)=\sum_{n_{1}, n_{2}=0}^{\infty}\left\{\phi_{n_{1} n_{2}}^{++}\left(x_{i}\right) \cos 2 \pi n_{1} y_{1} \cos 2 \pi n_{2} y_{2}+\phi_{n_{1} n_{2}}^{+-}\left(x_{i}\right) \cos 2 \pi n_{1} y_{1} \sin 2 \pi n_{2} y_{2}\right. \\
\left.+\phi_{n_{1} n_{2}}^{-+}\left(x_{i}\right) \sin 2 \pi n_{1} y_{1} \cos 2 \pi n_{2} y_{2}+\phi_{n_{1} n_{2}}^{--}\left(x_{i}\right) \sin 2 \pi n_{1} y_{1} \sin 2 \pi n_{2} y_{2}\right\}
\end{aligned}
$$


We will be particularly interested in Lagrangians which are invariant under some discrete gauge group, possibly embedded at some scale in a gauged U(1). In this case we can complement the geometrical action with an internal one belonging to the discrete gauge subgroup. Thus e.g., in the case of a U(1) symmetry and denoting their charge scalar by $q$, the boundary condition for the $\frac{\pi}{2}$ rotation is given by

$$
\phi\left(x_{i}, y_{1}, y_{2}\right)=e^{i q \alpha} \phi\left(x_{i},-y_{2}, y_{1}\right),
$$

for some real $\alpha$ which is the same for all of the particles involved in the $\mathrm{U}(1)$ symmetry. It is then easy to see that a solution exists for $q \alpha=2 k \frac{\pi}{4}$, where $k=0, \pm 1, \pm 2 \ldots$ Without loss of generality we fix $\alpha=\frac{\pi}{4}$ so that the charges are just even integers $q=2 k$ for scalars. In particular, for even $k$ the mode is given by: $\phi_{n_{1} n_{2}}^{++}= \pm \phi_{n_{2} n_{1}}^{++}, \phi_{n_{1} n_{2}}^{--}=\mp \phi_{n_{2} n_{1}}^{-}$, $\phi_{n_{1} n_{2}}^{+-}=\phi_{n_{1} n_{2}}^{-+}=0$. For odd $k$ it is given by $\phi_{n_{1} n_{2}}^{+-}= \pm i \phi_{n_{2} n_{1}}^{-+}, \phi_{n_{1} n_{2}}^{++}=\phi_{n_{1} n_{2}}^{--}=0$. In all of these cases the linearly independent Fourier modes can be confined to the positive $n_{1}, n_{2} \geq 0$ sector. Notice that the zero mode survives only for $k$ multiple of 4 .

Regarding the fermions we also start with the boundary conditions for the torus translations. Periodic or antiperiodic boundary conditions are taken into account by replacing $n$ with $\tilde{n}=n+\frac{1-z_{p}}{2}$ in the usual Fourier series. Under a general rotation Weyl spinors transform as $\psi(x, y) \longrightarrow e^{i \frac{\vec{q}}{2} \vec{\sigma}} \psi\left(x, y^{\prime}\right)$. Taking the $\frac{\pi}{2}$ rotation to be around the third axis we find:

$$
\psi\left(x_{i}, y_{1}, y_{2}\right) \longrightarrow\left(\begin{array}{cc}
e^{i \frac{\pi}{4}} & 0 \\
0 & e^{-i \frac{\pi}{4}}
\end{array}\right) \psi\left(x, y^{\prime}\right)
$$

If the internal global symmetry is a $\mathrm{U}(1)$, then the boundary condition for the orbifold rotation eq. (3.14) is:

$$
\left.\psi\left(x_{i}, y_{1}, y_{2}\right)\right)=e^{i q \alpha} e^{i \frac{\pi}{4} \sigma_{3}} \psi\left(x,-y_{2}, y_{1}\right)
$$

One finds that for $\alpha=1 / 4$ a solution exists only for odd $q$. Besides, it is always possible to confine all the independent Fourier modes to the positive $n_{1}, n_{2} \geq 0$ sector. For $q=1+8 k$ we obtain a 1-component $2 \mathrm{D}$ fermion in the massless sector, otherwise there is no zero mode for the fermion. To sum up, depending on its charge, each particle may or may not have a zero mode and also a non-vanishing contribution to the Casimir energy (i.e. it contributes only if it admits an expansion, that is, it has a KK tower). Let us recall that only massive KK excitations contribute to the Casimir potential, the possible zero modes do not. Hence the crucial feature in order to compute the potential is the presence or not of a KK tower for a given particle.

Finally, vector fields transform as $P A_{\mu}\left(x^{\sigma}\right) P^{-1}=\mathcal{P}_{\mu}{ }^{\nu} A_{\nu}\left(\mathcal{P}_{\rho}^{-1}{ }^{\sigma} x^{\rho}\right)$. Since they transform in the adjoint of their gauge group the orbifold action is given by:

$$
A_{\mu}\left(x_{i}, y_{1}, y_{2}\right)=U\left\{A_{i}\left(x_{i},-y_{2}, y_{1}\right),-A_{3}\left(x_{i},-y_{2}, y_{1}\right), A_{2}\left(x_{i},-y_{2}, y_{1}\right)\right\} U^{\dagger} .
$$

In the case of a U(1) symmetry, the U's cancel out. The key observation is that the boundary conditions project out the Wilson lines. As explained in the text, this is the main reason why we study orbifolds. Again, the most general solution is a combination 
of positive modes. Note that one can add however quantized Wilson line backgrounds of order 4 . We have checked that this extra discrete degree of freedom only gives rise to new Type $S$ vacua which are of no relevance for the present discussion.

Once we have found the expansion for each field, we insert it in the path integral, through the action, and proceed in the same way as in the torus or the circle. After integration in the compact coordinates, the only difference between $T^{2}$ and $T^{2} / Z_{4}$ is in the values that $\tilde{n}_{1}, \tilde{n}_{2}$ can take. We have seen that in $T^{2} / Z_{4}$ the independent normal modes can be confined to the positive $\tilde{n}_{1}, \tilde{n}_{2} \geq 0$ sector. Thus, the potential for the orbifold is given by the torus result eq. (3.8) evaluated at $\tau=i$ and $\theta_{1}=\theta_{2}=\theta$ and with the restriction $\tilde{n}_{1}, \tilde{n}_{2} \geq 0$ :

$$
V_{p}=(-1)^{2 s_{p}+1} n_{p} \frac{-i}{2} \sum_{n_{1}, n_{2}=0}^{\infty} \int \frac{d^{2} p}{(2 \pi)^{2}} \log \left[-p^{2}+\left(m_{p} \frac{H}{v}\right)^{2}+\frac{1}{a^{2}}\left(\tilde{n}_{2}^{2}+\tilde{n}_{1}^{2}\right)\right]
$$

Now, because we are at $\tau=i$, the modified indices $\tilde{n}_{1}, \tilde{n}_{2}$ appear only squared. This means that we can extend the summation in $n_{1}, n_{2}$ to the integers (remember that the zero mode, $n_{1}=n_{2}=0$, does not contribute to the Casimir energy and that $\tilde{n}=n+\theta$ ) as in the general formula of the torus. For this reason, we could just use the general formula, eq. (3.9) evaluated at $\tau=i$ and with an extra factor $\frac{1}{4}$.

For $\theta=0$ it is shown in appendix $\mathrm{B}$ that the contribution from a massless particle can be expressed in a simple form:

$$
V_{\mathcal{C}}[a, \tau=i, 0,0,0]=\frac{1}{(2 \pi a)^{2}} \frac{\mathcal{G}}{3},
$$

where

$$
\mathcal{G} \equiv \sum_{n=0}^{\infty} \frac{(-1)^{n}}{(2 n+1)^{2}}
$$

is Catalan's constant, $\mathcal{G} \simeq 0.915966$. For $\theta=\frac{1}{2}$, the Casimir energy reads

$$
V_{\mathcal{C}}\left[a, \tau=i, 0, \frac{1}{2}, \frac{1}{2}\right]=\frac{-1}{(2 \pi a)^{2}} \frac{\mathcal{G}}{6}=-\frac{1}{2} V_{\mathcal{C}}[a, \tau=i, 0,0,0] .
$$

The discussion for the massive case is essentially the same as for the segment. For $a^{-1} \ll m_{p}$ (the particle mass), the potential is exponentially suppressed with the mass, while for $a^{-1} \gg m_{p}$ it tends to $V_{\mathcal{C}}[a, \tau=i, 0, \theta, \theta]$, growing as $\propto a^{-2}$. In the case of $S^{1} / Z_{2}$ the potential for a massless particle decayed as $\propto R^{-6}$ and that is why $R^{6} V$ was constant in the massless limit and easier to plot. We had to look for the minima followed by zeros of $R^{6} V$, since only those meant a minima for $V$. Now we are concerned with the zeros of $a^{2} V$ as a function of $a$, since those are also zeros of $V$. In both cases we can normalize the potential in units of degrees of freedom. Finally, as in the case of the segment, fermions can be either periodic or antiperiodc. The only difference is that the factor $-\frac{7}{8}$ is now replaced by a factor $-\frac{1}{2}$. 


\subsection{Embedding $Z_{4}$ into discrete gauge symmetries}

From the discussion above, particularly eq.(3.20), one observes that if fermions are not charged under a discrete subgroup of a $\mathrm{U}(1)$ symmetry (i.e. $\alpha=0$ ) there is no KK tower for any fermions in the theory. Then the KK towers are purely bosonic and the Casimir potential becomes unbounded from below as $a^{2} \rightarrow 0$. This means we get a vacuum of Type $S$ which is consistent with AdS-phobia but leads to no constraint. In order to obtain Type $P$ vacua leading to interesting constraints on the SM we need to embed the $Z_{4}$ action into an order eight discrete subgroup of a gauge (or gaugable) U(1) symmetry, to match the $e^{i \pi / 4}$ phase that fermions get under a $\pi / 2$ rotation. In this way the SM fermions may get KK excitations and contribute to the Casimir energy, which is what we are searching for in order for neutrinos to play a role. We will consider three possibilities for such an embedding

a) Discrete subgroups of the Cartan subalgebra of the $\mathrm{SM}$ gauge group $\mathrm{SU}(3) \times \mathrm{SU}(2) \times$ $\mathrm{U}(1)$.

b) Discrete gauge symmetries. We will consider symmetries which are subgroups of U(1) global symmetries of the SM and may be gauged at a higher scale. This is the case of $(B-L)$ which is a global anomaly-free symmetry of the SM (if right-handed neutrinos are present) and is also a global gauge symmetry of the R-parity conserving MSSM. If the SM or MSSM is extended by a $\mathrm{U}(1)_{B-L}$ gauge symmetry, this embedding will always exist. However discrete $Z_{N}$ subgroups of $B-L$ may be gauged with no need for the full gauge group $\mathrm{U}(1)_{B-L}$ to be gauged at any scale, see e.g. [63, 64].

c) Discrete R-symmetries in the case of the SUSY SM. Those may appear in string compactifications as gauged R-symmetries, see e.g. [65-70].

The embeddings of the first type are always available and hence the conclusions one derives from the existence or not of AdS vacua in that case will always apply. For the other symmetries the conclusions will depend on whether the theory underlying the $4 \mathrm{D}$ string compactification leading to the SM admits those symmetries. In particular if $\mathrm{U}(1)_{B-L}$ is gauged, we will always be allowed to twist by any discrete subgroup of it.

For convenience of the reader, in table 1 we show the quantum numbers of SM or MSSM particles with respect to some relevant U(1) symmetries and R-symmetries (in the MSSM case). We will consider twisting with respect to discrete subgroups of them, as well as the Cartan subalgebra of $\mathrm{SU}(3) \times \mathrm{SU}(2)$.

\subsubsection{AdS SM $Z_{4}$ vacua}

It is easy to check that embedding $Z_{4}$ into a discrete subgroup (of order eight) of weak hypercharge $Y$ or the third component of weak isospin leads to uninteresting but safe Type $S$ vacua. Indeed the reader may check that only left-handed quarks and leptons build a KK tower and contribute in this case. Since the right-handed fermions are projected out, no masses can appear for the fermions. Thus the KK towers are dominated by these 


\begin{tabular}{|c|c|c|c|c|c|c|c|c|c|}
\hline Generator & $\mathrm{Q}$ & $\mathrm{u}$ & $\mathrm{d}$ & $\mathrm{L}$ & $\mathrm{e}$ & $\nu_{R}$ & $\tilde{H}$ & $\tilde{\tilde{H}}$ & $\tilde{g}, \tilde{W}, \tilde{B}$ \\
\hline $\mathrm{Y}$ & 1 & -4 & 2 & -3 & 6 & 0 & -3 & 3 & 0 \\
\hline $\mathrm{B}-\mathrm{L}$ & 1 & -1 & -1 & -3 & 3 & 3 & 0 & 0 & 0 \\
\hline $\mathrm{U}(1)_{s}$ & -1 & -1 & -1 & -1 & -1 & -1 & 1 & 1 & 1 \\
\hline
\end{tabular}

Table 1. Some fermion U(1) q-charges considered in the text. The line in the bottom is the R-symmetry of the MSSM and the corresponding sfermions have charge $q+1$.

massless fermions, which overwhelm the contributions of the photon and graviton avoiding AdS minima to develop.

On the other hand if we embed $Z_{4}$ into a discrete subgroup of the $\mathrm{SU}(3)$ colour Cartan subalgebra, Type $D$ minima develop. To see how this comes about, we begin by assigning periodic boundary conditions for the torus translations to all particles. Regarding the orbifold rotation boundary conditions, notice that all of the particles except for quarks and gluons have zero (strong) charge. From eqs. (3.18) and (3.20) this means that the Higgs bosons survive but the leptons are projected out. For the quarks the boundary condition is given by the following equation, diagonal in colour space:

$$
\Psi\left(x_{i}, y_{1}, y_{2}\right)=e^{i\left(q_{3} T_{3}+q_{8} T_{8}\right)} e^{i \frac{\pi}{4} \sigma_{3}} \psi\left(x,-y_{2}, y_{1}\right) .
$$

We know that a colour will survive only if its corresponding charge is odd. Introducing the Gell-Mann matrices we find these three equations: $q_{3}+q_{8}=$ odd,$-q_{3}+q_{8}=$ odd and $2 q_{8}=o d d$ for the three colours respectively. If the first two are satisfied then the third one is not satisfied, as can be seen by adding them. Thus, only two colours of quarks will get KK towers and no leptons. Doing the same for the gluons, one finds that $\mathrm{SU}(3)$ is broken to $\mathrm{SU}(2) \times \mathrm{U}(1)$. With this spectra, the contribution from the photon and graviton dominates down to the QCD transition, where the quarks make the potential positive. Thus, a minimum always develops, see figure 5(a). So, remarkably, the SM as it is would be excluded by the existence of this AdS vacuum. There is no dependence on any particle mass (like the lightest neutrino mass in the neutrino type of vacua) which could save the day. As we said, the reason why the SM necessarily has AdS vacua is that it is too fermionic. If the theory had more bosons than fermions this vacuum would become unstable and consistency with the WGC would be obtained. That is precisely what is naturally provided by a SUSY version of the SM which we consider next.

\section{$4 \quad Z_{4}$ MSSM vacua}

We now turn to the case of the MSSM. We consider here for definiteness the case of the simplest R-parity conserving version of the SM. Thus we have sfermions partners for fermions, Higgsinos for Higgses (we assume a minimal set) and gauge bosons come with gauginos. Let us check first whether twisting by discrete subgroups of the Cartan subalgebra of the SM lead to dangerous AdS vacua, as it happened in the non-SUSY SM. As in the latter, embedding the twist into hypercharge or weak isospin lead only to Type 


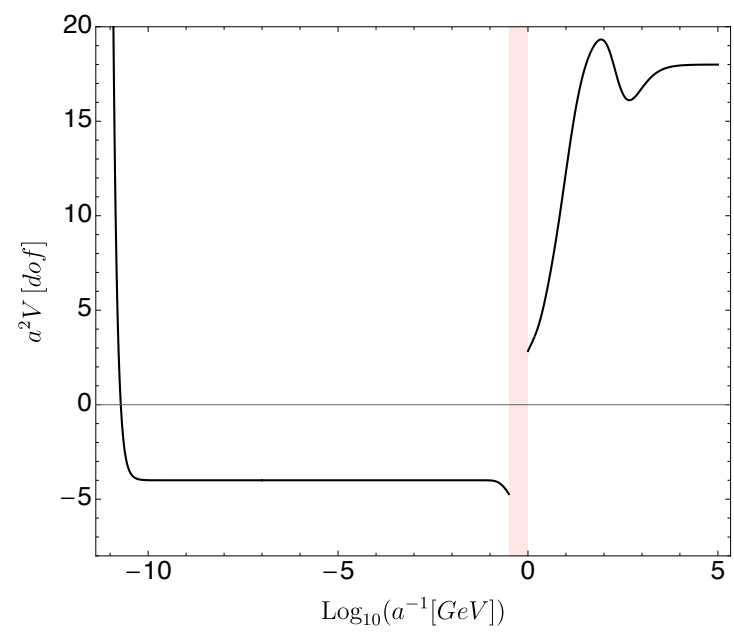

(a)

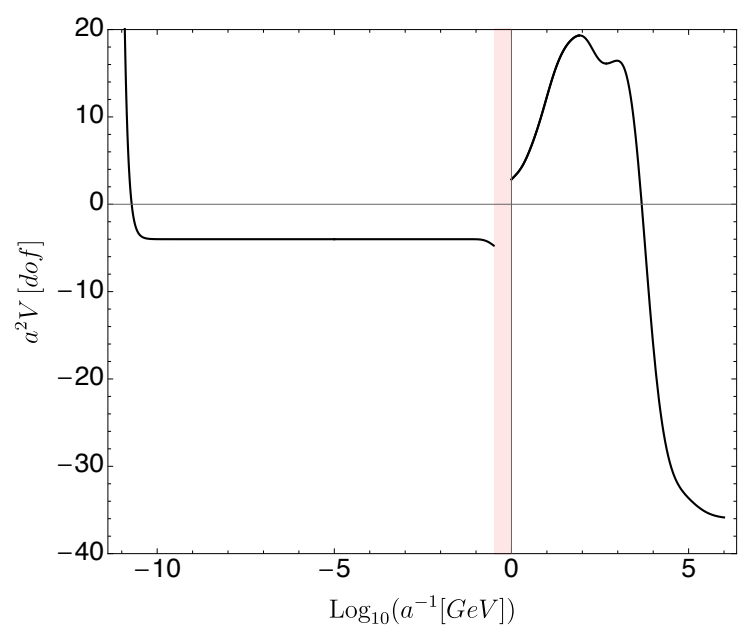

(b)

Figure 5. (a) Effective potential of the Standard Model compactified in $T^{2} / Z_{4}$ embedded into a discrete subgroup of the $\mathrm{SU}(3)$ colour Cartan subalgebra. All particles have periodic boundary conditions. As usual, the Higgs is fixed at its minimum. There is no runaway solution, so the minimum which appears around the QCD transition is of type D. The SM as it is appears to be inconsistent with Quantum Gravity. (b) The same plot but for the MSSM. The addition of extra bosonic degrees of freedom creates a runaway behaviour in the potential which could make the minima unstable. The MSSM would then appear to be consistent with the no stable AdS hypothesis.

$S$ vacua. If we embed $Z_{4}$ into the Cartan subalgebra of $\mathrm{SU}(3)$ the following particles get KK towers,

- Two colours of quarks $\rightarrow 48$ d.o.f.

- One colour of squarks $\rightarrow 24$ d.o.f.

- All sleptons $\rightarrow 24$ d.o.f.

- Gauge bosons, graviton and Higgs $\rightarrow 34$ d.o.f.

The contribution of fermions and sfermions cancel neatly. But altogether the number of bosons with a KK tower exceeds the number of fermions (since 8 gauginos do not get a KK tower, as well as Higgsinos). Therefore, as $a^{2} \rightarrow 0$ the potential necessarily develops an instability and the vacuum is of Type $S$, see figure 5(b). Thus the MSSM, due to the presence of the sparticles, survives the test that the SM failed.

The dimension 4 action of the R-parity conserving MSSM has two family-independent global symmetries, one being $B-L$, like in the SM. The other is an R-symmetry $\mathrm{U}(1)_{s}$ with charges shown in table 1 . Let us consider them in turn. 


\subsection{Embedding into a discrete subgroup of $\mathrm{U}(1)_{B-L}$}

As we said, an embedding of $Z_{N}$ into a discrete subgroup of $\mathrm{U}(1)_{B-L}$ will always be possible if the MSSM is extended to include a $\mathrm{U}(1)_{B-L}$ gauge symmetry. This symmetry is automatically present as a global symmetry in the R-parity conserving MSSM, and indeed the $B-L$ symmetry is gauged in a multitude of string semi-realistic models (see e.g. [71-74]). A discrete subgroup of $\mathrm{U}(1)_{B-L}$ may also be gauged without the full $\mathrm{U}(1)_{B-L}$ gauge symmetry surviving at low energies. For our purposes there is no practical difference although of course, if the symmetry $\mathrm{U}(1)_{B-L}$ is fully gauged in nature our conclusions below would be completely generic.

The quantum numbers of each MSSM particle with respect to $(B-L)$ are shown in table 1. Since the $q$ values shown in the table are all odd, all quarks and leptons get a KK tower, while sfermions do not. Gauginos and Higgsinos do not get KK towers since they do not have $B-L$ charge, whereas Higgs scalars do have a KK tower. The structure of the Casimir potential is, up to numerical factors with no important impact, practically identical to the potential that we depicted for the case of the circle with Wilson lines fixed. We show $a^{2} V(a)$ for this $Z_{4}$ case in figure 6(a). We find one dS and (possibly) one AdS minima at the neutrino scale, depending on the mass of the lightest Dirac neutrino. Since there are more fermionic than bosonic degrees of freedom the potential keeps growing. This ensures the stability of the neutrino vacuum, eliminating the runaway solution that appears for the circle or the torus, where Wilson lines are present. In figure 7 we plot the potential around the neutrino scale as a function of the lightest Dirac neutrino, assuming Normal Hierarchy. We find that there is no minimum if its mass is lower than $7.25 \mathrm{meV}$. For Inverse Hierarchy the bound is $m_{\nu_{3}} \leq 2.05 \mathrm{meV}$. Thus we recover the possibility of an AdS vacuum which is avoided for appropriate neutrino masses, and get back the predictions for the cosmological constant and the EW hierarchy. We conclude that in the MSSM extended by $\mathrm{U}(1)_{B-L}$ (or a discrete subgroup) one finds the attractive list of four predictions listed in the introduction. But this time without runaway directions that could spoil them.

Let us also note that in the SUSY case we do not have the freedom to set the torus boundary conditions for the leptons AP and $\mathrm{P}$ for the quarks. The reason is that gauginos (and the gravitino) couple to both quarks and leptons so that one cannot chose different torus boundary conditions for them. Thus the problematic AdS vacua present in 3D for the SM and shown in figure 4(b) is not present in the SUSY case. In this section 4 we are always taking periodic boundary conditions for all fermions. If all fermion boundary conditions were AP we would get uninteresting Type $S$ vacua.

\subsection{Embedding into the U(1) $)_{s}$ R-symmetry}

We finally consider the embedding of $Z_{4}$ into an order eight discrete subgroup of the $\mathrm{U}(1)_{s}$ R-symmetry in table 1. R-symmetries of this type are also abundant in string theory [65-70]. In some cases they are gauged in the $\sigma$-model sense, and their anomalies are cancelled by a Green-Schwarz mechanism, see e.g. [75-83]. They are eventually broken upon SUSY breaking by dimension $<4$ operators. Note that the table shows the charges of fermions, and that of scalars is obtained by adding $(+1)$ and hence is always even. It is 


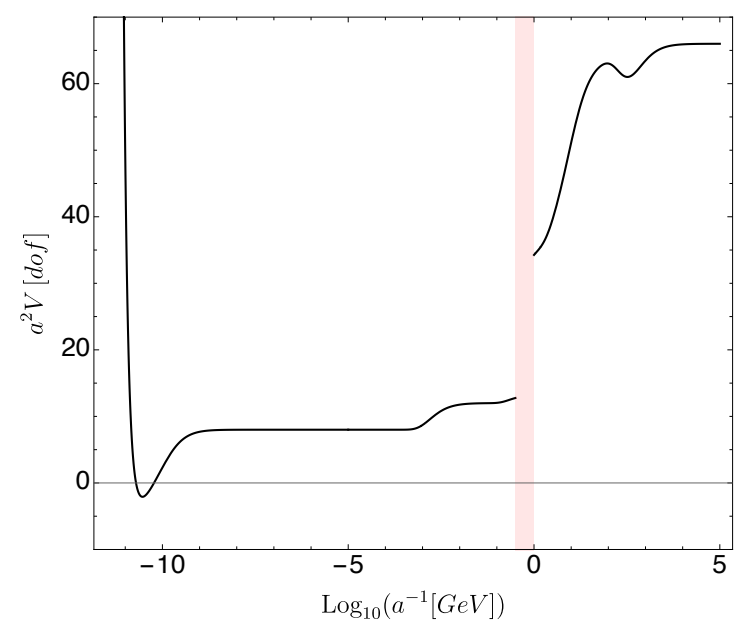

(a)

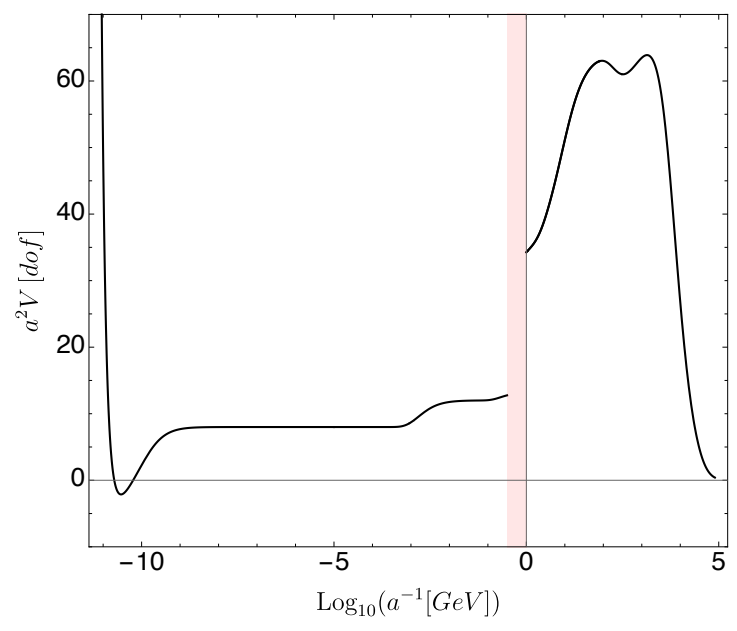

(b)

Figure 6. (a) Effective potential of the MSSM compactified in $T^{2} / Z_{4}$ embedded into $B-L$ and the Higgs fixed at its minimum. The only possible AdS minimum which may arise is the one associated with neutrino bounds. (b) Effective potential of the MSSM compactified in $T^{2} / Z_{4}$ and embedded into $\mathrm{U}(1)_{s}$. The Higgs is fixed at its minimum. The only possible minimum is the one associated with neutrino bounds. Recall that $2 \mathrm{D}$ minima only appear when $V(a)$ crosses zero. The neutrino minimum may be avoided (in both cases) if the lightest Dirac neutrino is light enough.

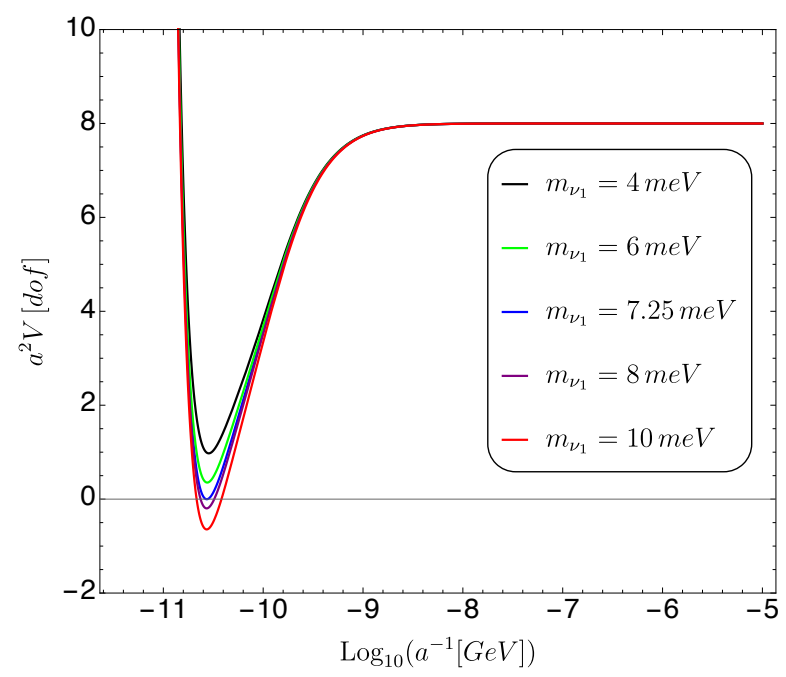

Figure 7. Zoom in of figure 6 around the neutrino scale. We always assume Dirac neutrinos, since Majorana neutrinos are ruled out. Using experimental constraints on the neutrino masses, we choose as free parameter the mass of the lightest neutrino. If its mass is larger than $7.25 \mathrm{meV}$, an AdS minima develops. 
easy to check that the complete spectrum of the MSSM get KK towers. In addition to the SM particles there will be contributions from KK towers of squarks, sleptons, gauginos, Higgsinos and the gravitino. We are thus including 48 (complex) scalars from the sfermions, 4 new Higgs (real)scalars, 4 Higgsinos, 12 gauginos and one gravitino. Altogether an addition of 60 net bosonic degrees of freedom which just balances the mostly fermion dominated SM. We show in figure 6(b) the function $a^{2} V(a)$ for this case, in which we have chosen a specific SUSY mass spectrum, provided in appendix D. The qualitative structure does not depend on the details of the spectrum. For values $1 / a \gtrsim M_{W}$, the SUSY thresholds open up, and there is a cancellation between fermions and bosons so that the potential goes to zero as $a \rightarrow 0$. Indeed we find that the leading contribution to the Casimir potential in this limit is given by

$$
V \rightarrow \frac{1}{(2 \pi a)^{2}} \frac{\mathcal{G}}{3}\left(\sum_{P}(-1)^{2 s_{p}+1} n_{P}\right)
$$

which cancels if all MSSM particles have KK towers. Here $\mathcal{G}$ is Catalan's constant. One has to be slightly more careful, though. Since the leading contribution as $a \rightarrow 0$ cancels, we have to examine the sub-leading terms to decide whether a runaway behavior exists or not. In the case of the segment one would have for a SUSY spectrum from eq. (2.19) a behaviour for small $R$

$$
V(R)_{R \rightarrow 0} \rightarrow \frac{1}{48 \pi R^{4}} \sum_{p}(-1)^{2 s_{p}} n_{p} m_{p}^{2} \equiv \frac{1}{48 \pi R^{4}} \delta_{S S}+\mathcal{O}\left(1 / R^{2}\right)
$$

where $\delta_{S S}$ is the supertrace over all masses. In the torus and $T^{2} / Z_{4}$ cases something analogous but slightly more complicated is obtained. The sub-leading mass-dependent contribution from a bosonic KK tower turns out to be (see appendix B)

$$
V^{(2)}[a, m, 0]=(a m)^{2}\left\{\pi \log (2 \pi m a)-\frac{\pi^{2}}{6}-\frac{5 \pi}{2}+\log \frac{\Gamma\left(\frac{1}{4}\right)}{2 \pi^{3 / 4}}\right\}
$$

and the opposite for a fermion. In the $(m a) \rightarrow 0$ limit we are considering the bosonic contribution is negative and the fermionic is positive. Numerically the situation is rather similar to case of the segment. In particular, the $\log (2 \pi m a)$ gives even more importance to the most massive particles in the spectrum with respect to the case when only the supertrace appeared. When it comes to understanding the final sign of the potential it must be noted that, if the sign of the supertrace is dictated by the most massive particle in the spectrum (as it is usually the case), it is guaranteed that the sign of the potential will be actually given by the one of the supertrace.

There are then essentially two model-dependent possibilities, depending on the particular structure of the SUSY spectrum:

- $\delta_{S S} \leq 0$. In this case there is again an instability at small $a$, and we have a Type $S$ vacuum. The WGC constraints are evaded and there is no inconsistency. 
- $\delta_{S S}>0$. In this case there is no runaway direction and the possibility of stable neutrino minima is recovered, as well as the corresponding predictions, we have a Type $P$ vacuum. This time not only the neutrino masses are constrained but also the SUSY masses.

Although both signs may lead to consistent theories, the $\delta_{S S}>0$ case is particularly attractive since the interesting constraints from the non-existence of neutrino AdS vacua are preserved. The sign of the superstrace is model dependent. In particular it depends on the values of the SUSY-breaking gaugino, squark, slepton and Higgssino/Higgs masses. One could think that in practically any MSSM constructed to date one has a dominant positive contribution to $\delta_{S S}$, since there are many more massive SUSY bosons than SUSY fermions, due to family replication. This is true, however, only if one restricts oneself to the effect of the SUSY partners of the observed SM particles. But here it is also relevant the mass of the gravitino $m_{3 / 2}$. The minimum SUSY breaking sector should involve at least a goldstone chiral multiplet, with a goldstino and a s-goldstino. The gravitino becomes massive combining with the goldstino and the s-goldstino typically also gets a mass of the same order. Thus one can also obtain $\delta_{S S}>0$ by having e.g. $m_{s g}$ heavier than all the rest of the spectrum. The sign also obviously depends on the possible presence of further particles beyond the MSSM and gravitino/goldstino sectors.

Still, the condition $\delta_{S S}>0$ may be an important constraint on specific SUSY extensions of the SM. In particular, consider a MSSM model in which the gluino is the heaviest SUSY particle, larger than all other MSSM sparticles but also larger than $m_{3 / 2}$ and $m_{s g}$. If the gluino is heavy enough one will violate the $\delta_{S S}>0$ condition and, although possibly consistent with the AdS-phobia condition, the radion potential would be unstable and the predictions from the neutrino AdS vacua would be lost. Note however that this can be avoided and the neutrino conditions would survive if e.g. the s-goldstino mass turns out to be heavier than the gluino. Since the s-goldstino and gravitino sector depends strongly on each model we cannot get a firm prediction that the heaviest observable SUSY particle should be a boson, since the SUSY-breaking is in general precluded from the observable sector. On the other hand $\delta_{S S}>0$ may be an interesting constraint to test in specific SUSY extensions of the SM.

\subsubsection{New AdS vacua for particular choices of SUSY spectra}

Although the $\delta_{S S}>0$ condition guarantees stability of the potential, it turns out that there can be new AdS minima at finite 1/a for particular choices of SUSY masses. Let us show here a couple of examples. The general structure of these examples requires three ingredients. First, to have a boson as the heaviest particle in order to fulfill the $\delta_{S S}>0$ condition and ensure local stability of the minimum. Second, to have an energy scale at which bosons dominate in order to be in the $V<0$ region and have a chance to form a minimum. Third, between this scale and the mass of the heaviest boson, we must have some fermionic degrees of freedom that can lift the potential to create the AdS minimum. To make things simple, we will consider here all the supersymmetric particles to have masses 


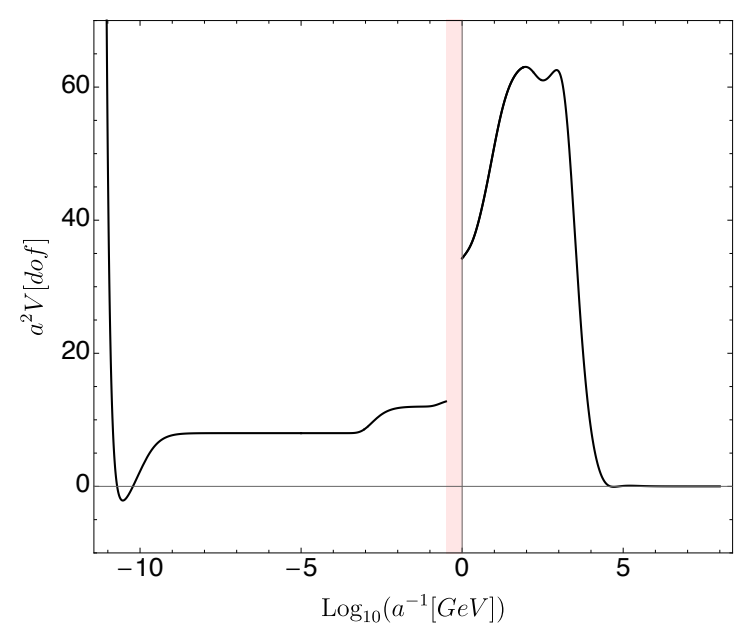

(a)

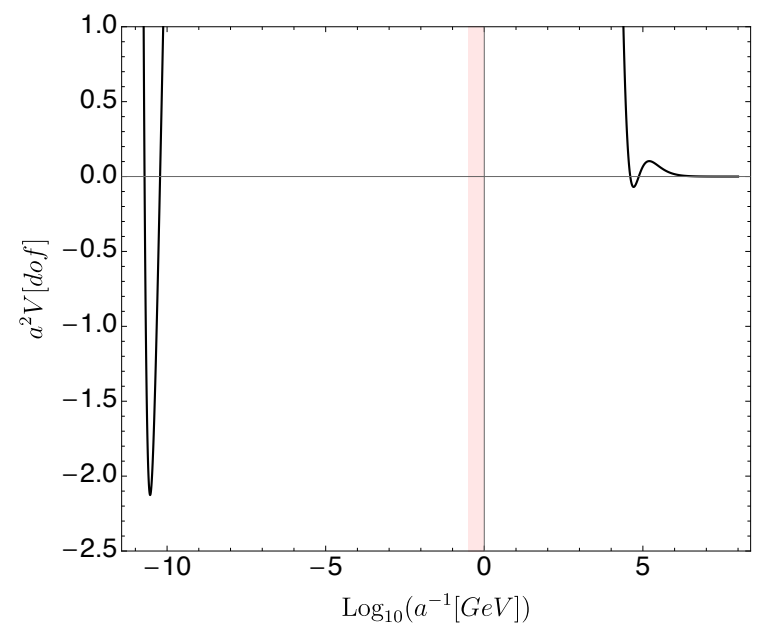

(b)

Figure 8. Effective potential of the Standard Model compactified in $T^{2} / Z_{4}$ and embedded into $\mathrm{U}(1)_{s}$. The masses of all superpartners of the SM particles are set to $1 \mathrm{TeV}$ except for the gravitino (4 d.o.f.), $m_{3 / 2}=19 \mathrm{TeV}$ and the s-goldstino (2 d.o.f.), $m_{\text {sgolds }}=33 \mathrm{TeV}$. (b) displays a zoom in of (a) and it can be seen that a Type P AdS minimum forms since it depends on the masses of the SUSY spectrum, hence giving some constraints from AdS-phobia.

around $1 \mathrm{TeV}$ except the boson that ensures $\delta_{S S}>0$ and the fermion that gives a positive contribution to the potential.

The first example of these kind of vacua is shown in figure 8 and we can constraint the relation between the mass of the gravitino and the mass of the s-goldstino (for fixed masses of all the other superpartners). In particular, an AdS minimum forms from $m_{3 / 2}=19 \mathrm{TeV}$ and $m_{\text {sgolds }}=33 \mathrm{TeV}$ onwards, excluding these kind of spectra in SUSY models with $\mathrm{U}(1)_{S}$ symmetries. Another example of these kind of vacua can be seen in figure 9, in which we consider the most massive particle to be a squark of mass $m_{\text {squark }}=23 \mathrm{TeV}$ and the fermions that lift the potential to be the gluinos, with mass $m_{\mathrm{g}}=18 \mathrm{TeV}$. From these masses on an AdS minimum forms and we can exclude these kind of spectra in the SUSY extensions of the SM.

More generally, given a SUSY version of the SM, computing the supertrace gives us information on the stability of the lower dimensional compactification and possible constraints on the SUSY spectrum and other parameters, i.e. neutrino masses and the cosmological constant. Furthermore one has to check whether additional AdS vacua can form depending on particular choices of SUSY masses.

\subsubsection{Charge-colour breaking AdS minima}

It is well known that the 4D MSSM has, apart from the standard EW minima in which the Higgs fields get a vev, a plethora of other possible minima in which squarks or sleptons are the ones who get a vev. [84, 85]. The space of these other minima is in general complicated and strongly dependent on the SUSY-breaking parameters. Many of these other minima are driven by the trilinear scalar couplings of the form e.g. $A_{t} h_{t}\left(\tilde{t}_{R} H_{u} \tilde{t}_{L}\right)+$ h.c., where $A_{t}$ 


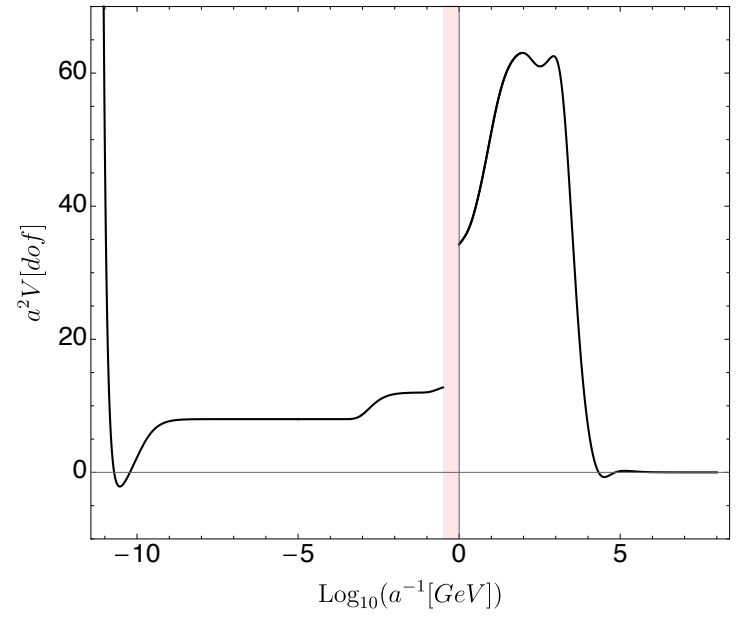

(a)

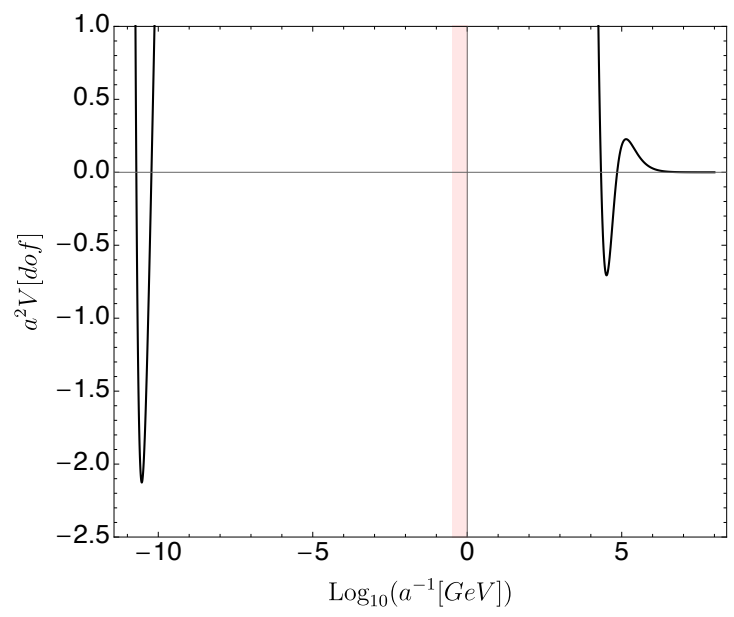

(b)

Figure 9. Effective potential of the Standard Model compactified in $T^{2} / Z_{4}$ and embedded into $\mathrm{U}(1)_{s}$. The masses of all superpartners of the SM particles are set to $1 \mathrm{TeV}$ except for the gluinos (16 d.o.f.), $m_{\mathrm{g}}=18 \mathrm{TeV}$ and 1 squark (12 d.o.f.), $m_{\mathrm{squark}}=23 \mathrm{TeV}$. (b) displays a zoom in of (a) and it can be seen that a Type P AdS minimum forms since it depends on the masses of the SUSY spectrum, hence giving some constraints from AdS-phobia.

is a soft parameter with dimension of mass, see [85] for a detailed analysis and references. One can derive necessary conditions on soft masses in order to avoid these minima to be lower than the standard Higgs minimum. A well known bound for minima derived from this trilinear stop coupling is

$$
\left|A_{t}\right|^{2}<3 h_{t}^{2}\left(m_{H_{u}}^{2}+m_{\tilde{t}_{L}}^{2}+m_{\tilde{t}_{L}}^{2}\right) .
$$

Weaker bounds may be derived by allowing minima lower than the Higgs one but imposing that the minimum is sufficiently stable at the cosmological level. In fact most of the examples of SUSY spectra discussed in the literature belong to this class in which the standard Higgs MSSM vacuum is metastable. These charge/colour breaking minima to which the Higgs vacuum is unstable to decay are AdS 4D minima, and the deepest of them will be stable. If we want to forbid AdS non-SUSY vacua altogether these 4D AdS minima should be absent from the start.

The logic of AdS-phobia then requires the Higgs vacua of the MSSM to be strictly stable and not just metastable. In fact full stability has been already advocated on different grounds, see e.g. [86] and references therein for a recent discussion. A general consequence of imposing strict stability and a Higgs mass around $125 \mathrm{GeV}$ is a quite heavy SUSY spectrum [86]. On the other hand this agrees well with the non-observation so far of any SUSY particle at LHC.

In going to $2 \mathrm{D}$ on $T^{2} / Z_{4}$, a necessary condition in order not to get any of these AdS vacua inherited is to impose as a strict condition the stability of the Higgs MSSM vacuum in $4 \mathrm{D}$. In addition, one expects that some of the stable vacua in $4 \mathrm{D}$ may become unstable in 2D. So the actual conditions on SUSY breaking mass parameters will be stronger than the 
parent 4D conditions. Thus one should impose that 1) the MSSM Higgs vacuum is stable in $4 \mathrm{D}$ against the decay into charge/colour breaking minima and 2) it remains stable against decay in 4D. Unfortunately these conditions can only be checked in a case by case basis.

Summarizing, with the SM embedded into a SUSY completion the AdS vacua which appeared in the non-SUSY SM become unstable and the theory is safe. The constraints for the neutrino masses, cosmological constant and EW hierarchy are recovered from the existence of a $Z_{4}$ vacua with action embedded into a discrete subgroup of $B-L$ in the R-parity preserving MSSM. Further constraints on the SUSY spectrum appear from a $Z_{4}$ embedding into a discrete subgroup of the R-symmetry present in the R-parity preserving MSSM, if the supertrace $\delta_{S S}>0$. Note that the latter constraint on the MSSM spectrum would only arise if the discrete subgroup of $\mathrm{U}(1)_{s}$ is a gauge symmetry of the underlying theory.

We see that the EW scale is bounded to be close to its experimental value in order to avoid the generation of stable $2 \mathrm{D}$ AdS neutrino generated vacua. On the other hand, avoiding 4D and/or 2D AdS charge-colour breaking minima typically requires relatively large SUSY masses in the multi-TeV region. Note that this could explain the so called little hierarchy problem: the SUSY spectrum needs to be relatively heavy in order to avoid these dangerous minima. On the other hand the EW scale is kept smaller by the condition that the lightest neutrino is sufficiently light to avoid AdS neutrino vacua. We must emphasize, though, that from this discussion the scale of SUSY breaking needs not be in the multi-TeV region. It could be much larger, up to a scale e.g. of order $10^{10} \mathrm{GeV}$ and the stability properties would still persist.

\subsection{Twisted sectors}

In all the previous sections we have not discussed the possible existence of twisted sectors. The presence of those is expected if there are orbifold fixed points in the compactification. Fixed points may be absent if the twist in the torus is accompanied by some translation in the compact six dimensions of the original $4 \mathrm{D}$ compactification. If they are present, in general there will be $2 \mathrm{D}$ particles from twisted sectors. Thus e.g. they explicitly arise in heterotic orbifold compactifications to 2D (see e.g. [87-93]). In Type II orientifolds there may be branes (D-strings in the 2D case) localized on the fixed points, leading to additional massless particles in the 2D theory. A full discussion of these twisted sectors would require a full knowledge of the underlying string compactification which leads to the SM or the MSSM in the first place. Fortunately for our purposes, to leading order we can ignore the effect of possible twisted sectors in our Casimir energy computations. This is because any twisted particle is localized in the singularities and hence does not have any KK tower. As we have emphasized, only states with KK towers contribute to the Casimir potential and hence twisted sectors do not modify to leading order the structure of the radius potential. In this respect their effect is similar to untwisted zero modes which also do not contribute to the Casimir potential. Notice however that in principle a radius independent constant contribution could arise from the twisted sector. This could be interpreted as a contribution of the tension of the objects localized in the singularities. Like e.g. in orientifold compactifications in which the tension of branes and 
orientifolds cancel, one may expect such local contribution to cancel at leading order. We are assuming that vacua exist in which such constant contribution is either absent or small. A small constant term would not alter significantly our discussion. At large $a$ the bulk 4D $\Lambda_{4}$ clearly dominates (it is multiplied by $a^{2}$ ) whereas at small $a$ the Casimir energy contributions grow rapidly like $1 / a^{2}$. Therefore, a constant piece may postpone the growth of a negative potential due to the photon and the graviton but it cannot avoid the appearance of an AdS minima.

The compactified models here discussed are in general chiral in 2D. Thus e.g. the model constructed by twisting by a $Z_{4}$ subgroup of $B-L$ have zero modes transforming like quarks under the SM group, with different $2 \mathrm{D}$ chiralities and has $\mathrm{SU}(2)_{L} 2 \mathrm{D}$ anomalies. The model obtained by twisting by a $\mathrm{U}(1)_{s}$ subgroup also has chiral zero modes transforming like all fermions of the MSSM. In 2D there are in general gauge and gravitational anomalies [94] and indeed these spectra by themselves have 2D anomalies. In particular, gauge anomalies are given by the quadratic Casimir eigenvalue $T_{a}$ of each chiral fermion, with sign depending on whether the zero mode is left- or right-moving. In principle one could use $2 \mathrm{D}$ anomaly cancellation conditions to try to figure out what the quantum numbers of the 2D twisted sectors could be. Indeed, one may easily obtain anomaly-free 2D theories by adding appropriate representations, which would be $2 \mathrm{D}$ lepton-like objects in our case. See e.g. [87] for specific 2D examples of anomaly cancellation. Although, as discussed above, the twisted sectors play no role in the computation of the Casimir potential, it would be interesting to study further the $2 \mathrm{D}$ anomaly constraints in specific $2 \mathrm{D}$ compactifications of the SM, MSSM or generalizations, i.e. compactifying a 10D theory directly to 2D. We leave this for future work.

\section{Conclusions and outlook}

In this paper we have studied compactifications of the SM to 3D and 2D, looking for stable AdS vacua, completing and generalizing previous work in $[39,41]$. In those works it was shown how the Casimir energy of the lightest sector of the SM gives rise to a radius dependent potential which may have AdS minima. Our motivation was the conjecture in [33] that posits that no theory with stable, non-SUSY AdS vacua can be embedded into a consistent theory of quantum gravity. In [39] constraints on neutrino masses were obtained from this condition applied to the SM compactified on the circle and on the torus. Here the assumption of background independence is crucial so that the constraints can be applied to the theory obtained upon compactification. This is also an important assumption in the present paper. For the minimal SM one finds that neutrinos cannot be Majorana (as in fact already suggested in [33]) and upper bounds on the lightest neutrino mass $m_{\nu_{1}} \leq 4.1 \times 10^{-3} \mathrm{eV}(\mathrm{NH})$ or $m_{\nu_{3}} \leq 1 \times 10^{-3} \mathrm{eV}(\mathrm{IH})$ are obtained. Furthermore, it was found that the $4 \mathrm{D}$ cosmological constant is bounded from below by the scale of neutrino masses, $m_{\nu_{1}}^{4} \lesssim \Lambda_{4}$. This is an attractive prediction, since it is the first argument implying a non-vanishing $\Lambda_{4}$ only on the basis of particle physics, with no cosmological input. Finally, the upper bound on the neutrino mass implies an upper bound on the EW scale (for fixed Yukawa coupling), eqs. (2.7), (2.8). This could give an explanation for the stability of the 
Higgs mass against quantum corrections, i.e. the hierarchy problem. Larger values of the EW scale allow for the generation of AdS vacua.

Although the AdS minima which may arise are perturbatively stable, non-perturbative instabilities could arise towards decay into lower minima or runaway directions, if present at smaller compact radius $R<1 / m_{e}$, with $m_{e}$ the electron mass [42]. Indeed, as we decrease $R$, new thresholds of leptons and quarks become relevant to the Casimir energy and the potential becomes more and more complex. In particular, it was shown that the presence of photon Wilson line moduli may give rise to non-perturbative instabilities [42]. If such instabilities exist, there would be no contradiction with AdS-phobia, although then we would lose the attractive constraints summarized above.

Searching for predictive vacua, in this paper we study SM compactifications in which Wilson line moduli of the SM gauge group are projected out. This is known to happen in orbifold $T^{2} / Z_{N}$ compactifications and we consider in particular the $Z_{4}$ case because of its simplicity and because for $N>2$ the complex structure of the torus is also projected out and we are left with a potential depending only on the area of the torus and the Higgs scalar. We classify the different vacua we find as Type $D$, which contain a stable AdS minimum which cannot be avoided by constraining free parameters of the theory, Type $S$, which have no stable AdS minimum and Type $P$, which have AdS vacua or not depending on some free parameters of the theory. Twisting only by $Z_{N}$ gives rise only to Type $S$ vacua and no constraints. More interesting vacua are obtained if we embed the $Z_{4}$ symmetry into $\mathrm{U}(1)$ gauge (or gaugable) degrees of freedom of the theory. Embedding $Z_{4}$ into the SM gauge group we obtain necessarily some stable AdS vacua for the SM. Thus, remarkably, the SM as it stands would not be embeddable into a consistent theory of quantum gravity. This is true for any value of neutrino masses. Note that a further problem for the minimal $\mathrm{SM}$ is the possible existence of a second $4 \mathrm{D}$ high energy Higgs vaccum at scales above $10^{10} \mathrm{GeV}$. This minimum would be AdS and, if stable, would again be in contradiction with the OV conjecture.

Interestingly, if the SM is embedded into a SUSY version like the MSSM, those AdS vacua become automatically unstable and the theory is consistent with WGC constraints. Furthermore, the second high energy Higgs vacuum also disappears if the SUSY breaking scale is not above $\sim 10^{12} \mathrm{GeV}$. We study further possible Type $P$ vacua of the MSSM compactified in $T^{2} / Z_{4}$ which could lead to constraints on particle physics. In particular, an interesting vacuum is obtained if we embed $Z_{4}$ into a discrete subgroup of the $\mathrm{U}(1)_{B-L}$ symmetry, which is a global symmetry of the R-parity conserving MSSM and may be gauged at higher energies. The resulting vacuum has a potential with just a possible AdS minimum around the neutrino region. This is the same AdS minimum found in [39, 41], and may be avoided if one of the (Dirac) neutrinos is sufficiently light. Thus we again have the constraints on the cosmological constant and the gauge hierarchy described in $[39,40]$. This suggests that the MSSM should be extended to include a $\mathrm{U}(1)_{B-L}$ gauged symmetry.

It is interesting to see whether there are other $Z_{4}$ compactifications leading to further phenomenological restrictions e.g., on the values of the SUSY masses. The other familyindependent global symmetry of the R-parity preserving MSSM is the R-symmetry U $(1)_{s}$ of table 1. Such type of R-symmetries are gauged in the $\sigma$-model sense in $N=1$ supergravity 
theories obtained in string compactifications. If we embed $Z_{4}$ into a discrete subgroup of this $\mathrm{U}(1)_{s}$ we obtain a 2D model in which all MSSM particles have a KK tower. In this case, depending (essentialy) on the mass ${ }^{2}$ supertrace, new conditions on the SUSY spectrum appear. However in this latter case the constraints would apply only if the underlying theory contains such discrete R-symmetry.

Additional constraints come from avoiding the presence of charge and/or colour breaking AdS minima both in 4D and 2D. This typically requires a relatively heavy SUSY spectrum in the multi-TeV region [86]. These arguments could provide a possible explanation for the little hierarchy problem, i.e. the fact that, if low energy SUSY is correct, the SUSY particles seem to be relatively heavy compared to the EW scale. From the present point of view the EW scale is small due to the constraint in eqs.(2.7), (2.8) whereas the SUSY spectrum is forced to be relatively large to avoid charge/colour breaking AdS minima.

An important question is whether the scale of SUSY-breaking $M_{S S}$ and the masses of SUSY particles are close to the EW scale and LHC energies or not. It would be extremely interesting if the AdS-phobia condition as applied to the MSSM could give us a hint on what the scale of SUSY breaking is. In particular it is conceivable e.g. that avoiding the appearance of AdS vacua when compactifying the MSSM down to 2D could require a lowenergy SUSY spectrum around a few TeV, low-energy SUSY. Or else that avoiding such vacua could require a very massive SUSY spectrum. Note in this respect that, as we said, SUSY cannot be arbitrarily high, since if $M_{S S}>10^{10} \mathrm{GeV}$ the second lower SM Higgs minimum may develop, which would be in AdS. To test whether there is a preference for low-energy SUSY or not coming from AdS-phobia we have to improve the present analysis. In particular we should consider the renormalization group improved couplings and masses. When going to very high energies (or rather very small $a$ ) large logs will appear which cannot be ignored at the quantitative level. The soft masses run and e.g the sign of $\delta_{S S}$ may change as masses and couplings run. We leave this important analysis for future work.

We have used the 2D vacua in this paper as auxiliary tools in order to derive constraints on the parent SM or MSSM 4D model. However some of these 2D vacua could be cosmologically interesting in the following sense. Consider the $2 \mathrm{D}$ vacua obtained from embedding into $\mathrm{U}(1)_{s}$. It contains zero modes for all the particles in the MSSM except for the Higgs bosons. Once the AdS neutrino (and/or SUSY) bounds are respected, the potential is monotonously decreasing into large $2 \mathrm{D}$ volume, the KK towers become massless and we recover the 4D MSSM (including the Higgs bosons) as $a \rightarrow \infty$. So one could speculate that the universe started two-dimensional and became $4 \mathrm{D}$ well before experimentally constrained cosmological events took place. In such a model the $4 \mathrm{D}$ cosmological constant would have been $2 \mathrm{D}$ volume dependent. The situation would be consistent with the conjecture in [32] that no stable dS vacuum should exist and the universe should have a runaway behavior. It would be interesting to explore whether a sensible cosmology could be constructed in this scheme.

Since the casuistic above could confuse the reader, let us conclude with a brief list our findings:

- The SM as it stands necessarily has stable AdS vacua in $2 \mathrm{D}$ and hence would be in the swampland. 
- In the MSSM those AdS vacua become unstable and lead to no incompatibility with quantum gravity.

- If the MSSM is extended by a $\mathrm{U}(1)_{B-L}$ gauge group (or a discrete subgroup), the theory has 2D AdS vacua which can be avoided if neutrinos are Dirac and the lightest is sufficiently light. The four predictions listed in the introduction are recovered.

- If in addition the MSSM 4D vacua has a gauged discrete R-symmetry, subgroup of the global U(1) $)_{s}$ R-symmetry of the R-parity preserving MSSM, further constraints on the SUSY spectra, depending on the supertrace, are obtained

- The hierarchy problem is solved by imposing absence of AdS neutrino vacua. But SUSY is needed to avoid additional AdS minima which would otherwise be present. Thus the SUSY spectrum could be substantially above the EW scale, but also possibly in the multi- $\mathrm{TeV}$ region.

Finally, it would be important to improve our understanding of the stability of this kind of SM compactifications to lower dimensions. In particular in the $2 \mathrm{D}$ vacua the radius $a$ does not propagate and the Higgs is the only propagating degree of freedom (along with the sfermions if present). It would be interesting to study how the tunneling towards lower minima or runaway directions happens in this class of theories. More generally the results depend on the validity of the assumption of the Ooguri-Vafa conjecture of AdS-phobia and it would be important to bring additional evidence in favour or against it. In the meantime we think it is well motivated to study what the consequences of its validity would be, and they look, indeed, quite intriguing.

\section{Acknowledgments}

We thank A. Font, F. Marchesano, V. Martin-Lozano, A. Uranga, I. Valenzuela, C. Vafa and especially M. Montero for useful discussions and suggestions. This work has been supported by the ERC Advanced Grant SPLE under contract ERC-2012-ADG-20120216320421, by the grant FPA2012-32828 from the MINECO, and the grant SEV-2012-0249 of the "Centro de Excelencia Severo Ochoa" Programme. A.H. is supported by the Spanish FPU Grant No. FPU15/05012 and E.G. by the Spanish FPU Grant No. FPU16/03985. 


\section{A One-loop effective potential in a $S^{1}$ compactification of the Standard Model}

We denote the quantum effective action by $\Gamma$ and the classical, background fields by an overline. We are interested only in the ground state of the theory. For this reason we can set the classical, background fields of the fermions to zero from the start. We perform the computation in the background-field gauge, because we will use the background-field method to compute the effective action and this choice enables us to maintain gauge invariance in the background, gauge-boson fields. If we parameterize the Higgs doublet as $\frac{1}{\sqrt{2}} \eta+\frac{1}{\sqrt{2}}\left(\begin{array}{l}1 \\ \mathrm{v}\end{array}\right)$, then the gauge fixing term is given by:

$$
\begin{aligned}
\mathcal{L}_{\text {Gauge Fixing }}= & -\frac{1}{2 \xi}\left[\partial_{\mu} W_{i}^{\mu}+g \varepsilon_{i j k} \bar{W}_{\mu j} W^{\mu k}+i \frac{\xi}{2} g\left(\eta^{\dagger} \frac{\sigma_{i}}{2} \mathrm{v}-\mathrm{v}^{\dagger} \frac{\sigma_{i}}{2} \eta\right)\right]^{2} \\
& -\frac{1}{2 \xi}\left[\partial_{\mu} B^{\mu}+\frac{i \xi g^{\prime}}{4}\left(\eta^{\dagger} Y \mathrm{v}-\mathrm{v}^{\dagger} Y \eta\right)\right]^{2}-\frac{1}{2 \alpha}\left[\partial_{\mu} G_{a}^{\mu}+g f_{a b c} \bar{G}_{\mu b} G^{\mu c}\right] .
\end{aligned}
$$

We work in the unitary gauge, obtained by taking the limit $\xi, \alpha \rightarrow \infty$. Using the available background gauge invariance one can gauge away all components except the one along the compact direction of those bosons in the Cartan Subalgebra of $\mathrm{SU}(3)_{C} \times \mathrm{SU}(2)_{L} \times$ $\mathrm{U}(1)_{Y}$. We denote these four Wilson lines as $\bar{Z}, \bar{A}, \bar{G}_{1}, \bar{G}_{2}$, corresponding to the vevs of the $\mathrm{Z}$ boson, the photon and the gluons associated with the two diagonal Gell-Mann matrices. We define the Wilson lines so that they absorb the gauge coupling constants in the covariant derivative. Using the background field method, we must compute the following path integral, neglecting terms associated with diagrams which are not connected and 1PI. At one-loop order we need only study the terms quadratic in the quantum fields, since linear terms would never give 1PI diagrams and zero order terms correspond to the classical (tree level) action. Denoting by $\Psi$ to all fermions and by $\eta$ to all the necessary ghosts, and denoting in general as $\phi$ to all fields in the theory, the measure would then be $D \phi=D g_{\mu \nu} D A_{\mu} D Z_{\mu} D W_{\mu}^{+} D W_{\mu}^{-} D H D \bar{\Psi} D \Psi D \bar{\eta} D \eta$ and the path integral to compute

$$
e^{i \Gamma\left[\bar{R}, \bar{H}, \bar{Z}, \bar{A}, \bar{G}_{1}, \bar{G}_{2}\right]}=\int D \phi e^{i S[\phi+\bar{\phi}]} .
$$

1PI, Connected

For completeness, we will write in some detail the SM Lagrangian. We will omit from the Lagrangian the part of the gauge-fixing term (A.1) that depends on the background fields (of course, it must included in the calculations of the effective action). We do not write in any detail the ghost Lagrangian $\mathcal{L}_{\mathrm{FP}}^{\mathrm{SM}}$, since it was shown in [49] that they do not contribute to the effective action at one-loop order. The computation of the effective potential of the Einstein-Hilbert Lagrangian compactified in $S^{1}$, to one-loop order, was performed in [49], so we also omit the details here. We will also omit the details of the counterterm Lagrangian and the renormalization procedure. To regularize we will use either Dimensional Regularization with extra flat dimensions or Zeta Function Regularization techniques. Note that $\mu, \nu, n, m$ space-time indices run from $0,1,2,3$; $i, j$ space-time indices from $0,1,2 ; i, j$ colour indices run from $1,2,3$ and $a, b$ colour indices run from $1,2 \ldots 8$. We will replace all space-time covariant derivatives by par- 
tial derivatives from the start, since we will be interested only in the one-loop corrections to the tree level potential. Finally, $f$ denotes sum over all fermions and $A$ sum over the fermions families: $e_{A}=(e, \mu, \tau),\left(\begin{array}{l}p_{A} \\ n_{A}\end{array}\right)=\left(\begin{array}{lll}u & c & t \\ d & s & b\end{array}\right)$.

$$
\begin{aligned}
& \mathcal{L}=\mathcal{L}_{\text {asymptotic }}^{\mathrm{SM}}+\mathcal{L}_{\text {basic interaction }}^{\mathrm{SM}}+\mathcal{L}_{\mathrm{FP}}^{\mathrm{SM}}+\mathcal{L}_{\text {counterterms }}^{\mathrm{SM}}+\mathcal{L}_{\text {Einstein-Hilbert }}+\Lambda_{4} \\
& \mathcal{L}_{\text {asymptotic }}^{\mathrm{SM}}=\mathcal{L}_{\text {Fermions }}+\mathcal{L}_{\mathrm{YM}}^{(2)}+\mathcal{L}_{\mathrm{SBS}}^{(2)} \\
& \mathcal{L}_{\text {Fermions }}=\sum_{f} \bar{\Psi}_{f}\left(i \tilde{\gamma}^{\mu} \partial_{\mu}-m_{f}\right) \Psi_{f} \quad \tilde{\gamma}^{\mu}=e_{n}^{\mu} \gamma^{n} \quad g_{\mu \nu}=\eta_{n m} e_{\mu}^{n} e_{\nu}^{m} \\
& \mathcal{L}_{\mathrm{YM}}^{(2)}=W_{\mu}^{-}\left[\eta^{\mu \nu} \partial^{2}-\partial^{\mu} \partial^{\nu}+\eta^{\mu \nu} M_{W}^{2}\right] W_{\nu}^{+}+\frac{1}{2} Z_{\mu}\left[\eta^{\mu \nu} \partial^{2}-\partial^{\mu} \partial^{\nu}+\eta^{\mu \nu} M_{Z}^{2}\right] Z_{\nu} \\
& +\frac{1}{2} A_{\mu}\left[\eta^{\mu \nu} \partial^{2}-\partial^{\mu} \partial^{\nu}\right] A_{\nu}+\frac{1}{2} G_{\mu}^{a}\left[\eta^{\mu \nu} \partial^{2}-\partial^{\mu} \partial^{\nu}\right] \delta_{a b} G_{\nu}^{b} \\
& \mathcal{L}_{\text {SBS }}^{(2)}=-\frac{1}{2} H\left[\partial^{2}+m_{H}^{2}\right] H \\
& \mathcal{L}_{\text {basic interaction }}^{\mathrm{SM}}=\mathcal{L}_{\mathrm{NC}}+\mathcal{L}_{\mathrm{CC}}+\mathcal{L}_{\mathrm{YM}}^{3}+\mathcal{L}_{\mathrm{YM}}^{4}+\mathcal{L}_{\mathrm{SBS}}^{(3+4)}+\mathcal{L}_{\mathrm{YW}} \\
& \mathcal{L}_{\mathrm{NC}}=\sum_{f} \bar{\Psi}_{f} \tilde{\gamma}^{\mu}\left(e Q_{f} A_{\mu}+\frac{g}{c_{W}}\left(g_{L}^{f} P_{L}+g_{R}^{f} P_{R}\right) Z_{\mu}+g_{s} G_{\mu}^{a} T_{f}^{a}\right) \Psi_{f} ; \\
& T_{f}^{a}=\left\{\begin{array}{lr}
0 & \text { colour singlet } \\
\frac{\lambda^{a}}{2} & \text { colour triple }
\end{array}\right\} \\
& \mathcal{L}_{\mathrm{CC}}=\frac{g \tilde{\gamma}^{\mu}}{\sqrt{2}} \sum_{A} \bar{\nu}_{A} W_{\mu}^{+} P_{L} e_{A}+\bar{p}_{A} W_{\mu}^{+} P_{L} n_{A}+h . c \\
& \mathcal{L}_{\mathrm{YM}}^{3}=i g c_{w}\left[\left(\partial_{\mu} W_{\nu}^{-}-\partial_{\nu} W_{\mu}^{-}\right) W^{+\mu} Z^{\nu}-h . c\right]-i e\left[\left(\partial_{\mu} W_{\nu}^{-}-\partial_{\nu} W_{\mu}^{-}\right) W^{+\mu} A^{\nu}-h . c\right] \\
& +i g c_{w}\left(\partial_{\mu} Z_{\nu}-\partial_{\nu} Z_{\mu}\right) W^{+\mu} W^{-\nu}-i e\left(\partial_{\mu} A_{\nu}-\partial_{\nu} A_{\mu}\right) W^{+\mu} W^{-\nu} \\
& -\frac{1}{2} g_{s} f_{a b c}\left(\partial_{\mu} G_{\nu}^{a}-\partial_{\nu} G_{\mu}^{a}\right) G^{\mu b} G^{\nu c} \\
& \mathcal{L}_{\mathrm{YM}}^{4}=-g^{2} c_{w}^{2}\left[W_{\mu}^{-} W^{+\mu} Z_{\nu} Z^{\nu}-W_{\mu}^{-} W_{\nu}^{+} Z^{\mu} Z^{\nu}\right]-e^{2}\left[W_{\mu}^{-} W^{+\mu} A_{\nu} A^{\nu}-W_{\mu}^{-} W_{\nu}^{+} A^{\mu} A^{\nu}\right] \\
& +e g c_{w}\left[2 W_{\mu}^{-} W^{+\mu} Z_{\nu} A^{\nu}-W_{\mu}^{-} W_{\nu}^{+}\left(Z^{\mu} A^{\nu}+A^{\mu} Z^{\nu}\right)\right] \\
& +g^{2}\left(W_{\mu}^{-} W^{+\mu} W_{\nu}^{-} W^{+\nu}-W_{\mu}^{+} W^{+\mu} W_{\nu}^{-} W^{-\mu}\right)+\frac{1}{4} g_{s}^{2} f_{a b c} f_{a d e} G_{\mu}^{b} G_{\nu}^{c} G^{\mu d} G^{\nu e} \\
& \mathcal{L}_{\mathrm{SBS}}^{(3+4)}=\frac{g^{2}}{4}\left[2 \mathrm{v} H+H^{2}\right]\left[W_{\mu}^{-} W^{+\mu}+\frac{1}{2 c_{w}^{2}} Z_{\mu} Z^{\mu}\right]-\lambda \mathrm{v} H^{3}-\frac{\lambda H^{4}}{4} \\
& \mathcal{L}_{\mathrm{YW}}=-\sum_{f} m_{f} \frac{H}{\mathrm{v}} \bar{\Psi}_{f} \Psi_{f} . \\
& g_{L, R}^{f}=T_{3 L, R}^{f}-s_{w}^{2} Q_{f}
\end{aligned}
$$

Taking into account the boundary conditions, each field is expanded in a Fourier series, as explained in the text. Since we are staying at cuadratic order, the integral over each field will be gaussian. Each gaussian integral reduces to computing the determinant of a certain operator. Using $\operatorname{det} A=e^{\log \operatorname{Tr} A}$, we transform the determinant into a trace. After computing the traces for the different particles one finds that the contribution of each particle to the Casimir potential can always be written as:

$$
V_{p}=(-1)^{2 s_{p}+1} n_{p} \frac{-i}{2} \sum_{n=-\infty}^{\infty} \int \frac{d^{3} p}{(2 \pi)^{3}} \log \left(-p^{2} \varphi^{2}+\left(m_{p} \frac{H}{\mathrm{v}}\right)^{2}+(n+\theta)^{2} \varphi^{2}\right) .
$$




\begin{tabular}{|c|c|c|c|}
\hline & $Q_{f}$ & $g_{L}^{f}$ & $g_{R}^{f}$ \\
\hline$\nu_{e}, \nu_{\mu}, \nu_{\tau}$ & 0 & $\frac{1}{2}$ & 0 \\
\hline$e, \mu, \tau$ & -1 & $-\frac{1}{2}+s_{w}^{2}$ & $s_{w}^{2}$ \\
\hline$u, c, t$ & $\frac{2}{3}$ & $\frac{1}{2}-\frac{2}{3} s_{w}^{2}$ & $-\frac{2}{3} s_{w}^{2}$ \\
\hline$d, s, b$ & $-\frac{1}{3}$ & $-\frac{1}{2}+\frac{1}{3} s_{w}^{2}$ & $\frac{1}{3} s_{w}^{2}$ \\
\hline
\end{tabular}

Table 2. $\mathrm{SU}(2)_{L} \times \mathrm{U}(1)_{Y}$ quantum numbers of the particles in the Standard Model.

\section{B One-loop effective potential in $T^{2} / Z_{4}$}

The first steps of the computation are essentially the same as in the previous appendix. Besides, some details were given in the text so we do not repeat them here. In the text we also mentioned that, to gain a better understanding of the Casimir potential, it is interesting to study the ultraviolet behaviour. In this appendix we will use the expressions from the text to extract this UV behaviour. To take this $a m \rightarrow 0$ limit, it will be useful to rewrite the general formula for the Casimir potential in the torus using the following formulas from $[95]$

$$
\operatorname{Li}_{s}\left(r e^{i \phi}\right)=\mathrm{Li}_{s}(r, \phi)+i\left[\operatorname{Ti}_{s}\left(\frac{r \sin \phi}{1-r \cos \phi}\right)-\mathrm{Ti}_{s}\left(\frac{r \sin \phi}{1-r \cos \phi}, \tan \phi\right)\right], \quad s=2,3
$$

where

$$
\begin{aligned}
\operatorname{Li}_{2}(r, \phi) & =-\frac{1}{2} \int_{0}^{r} \frac{\log \left(1-2 x \cos \phi+x^{2}\right)}{x} d x, \\
\operatorname{Li}_{3}(r, \phi) & =\int_{0}^{r} \frac{\operatorname{Li}_{s}(x, \phi)}{x} d x,
\end{aligned}
$$

and $\mathrm{Ti}_{s}$ are generalized inverse tangent integrals which verify: $\operatorname{Ti}_{s}(-x)=-\mathrm{Ti}_{s}(x)$, $\operatorname{Ti}_{s}(-x,-y)=-\operatorname{Ti}_{s}(x, y)$. Thus, $\operatorname{Li}_{s}\left(r e^{i \phi}\right)+\operatorname{Li}_{s}\left(r e^{-i \phi}\right)=2 \mathrm{Li}_{s}(r, \phi)$. Introducing this last equation in (3.9) we find:

$$
\begin{aligned}
& V_{\mathcal{C}}\left[a, t, m_{p}, \theta_{1}, \theta_{2}\right]=\frac{1}{(2 \pi a)^{2}}[ 2 a^{2} t_{2} m^{2} \sum_{p=1}^{\infty} \frac{\cos 2 \pi p \theta_{1}}{p^{2}} K_{2}\left(\frac{2 \pi p a m}{\sqrt{t_{2}}}\right) \\
&\left.+\frac{1}{2 \pi t_{2}} \sum_{n=-\infty}^{\infty}\left\{-\log r_{n} \operatorname{Li}_{2}\left(r_{n}, \phi_{n}\right)+\operatorname{Li}_{3}\left(r_{n}, \phi_{n}\right)\right\}\right], \\
& e^{\sigma_{+}}=e^{-2 \pi \sqrt{\left(n+\theta_{1}\right)^{2} t_{2}^{2}+m^{2} a^{2} t_{2}}} e^{i 2 \pi\left[-\left(n+\theta_{1}\right) t_{1}+\theta_{2}\right]}=r e^{i \phi} .
\end{aligned}
$$


Secondly, we set $t_{1}=0$ and $t_{2}=1$ in eq. (B.4) and eq. (B.5), $\theta_{1}=\theta_{2} \equiv \theta$, and take the massless limit:

$$
\begin{aligned}
V_{\mathcal{C}}[a, \theta]=\frac{1}{(2 \pi a)^{2}}[ & \frac{1}{\pi^{2}} \sum_{p=1}^{\infty} \frac{\cos 2 \pi p \theta}{p^{4}} \\
& \left.+\frac{1}{2 \pi} \sum_{n=-\infty}^{\infty}\left\{2 \pi|n+\theta| \operatorname{Li}_{2}\left(e^{-2 \pi|n+\theta|}, 2 \pi \theta\right)+\operatorname{Li}_{3}\left(e^{-2 \pi|n+\theta|}, 2 \pi \theta\right)\right\}\right] .
\end{aligned}
$$

Next, we can use that for periodic boundary conditions $\operatorname{Li}_{s}(r, 0)=\operatorname{Li}_{s}(r)$, and for antiperiodic $\operatorname{Li}_{s}(r, \pi)=\operatorname{Li}_{s}(-r)$. Finally, using the definition of the polylogarithm $\operatorname{Li}_{s}(z)=$ $\sum_{p=1}^{\infty} \frac{z^{p}}{p^{s}}$ we can do the geometric sums in $n$ using

$$
\begin{gathered}
\sum_{n=-\infty}^{\infty}|n|\left(e^{-2 \pi p}\right)^{|n|}=\frac{2 e^{-2 \pi p}}{\left(1-e^{-2 \pi p}\right)^{2}}=\frac{1}{2} \operatorname{csch}^{2} \pi p \\
\sum_{n=-\infty}^{\infty}\left(e^{-2 \pi p}\right)^{|n|}=\frac{1+e^{-2 \pi p}}{1-e^{-2 \pi p}}=\operatorname{coth} \pi p
\end{gathered}
$$

for the periodic case and

$$
\begin{aligned}
\sum_{n=1}|2 n+1|\left(e^{-2 \pi p}\right)^{|2 n+1|} & =\frac{2\left(e^{-6 \pi p}+e^{-2 \pi p}\right)}{\left(1-e^{-4 \pi p}\right)^{2}}=\operatorname{csch} 2 \pi p \operatorname{coth} 2 \pi p \\
& =\frac{1}{4} \operatorname{csch}^{2} \pi p+\frac{1}{4} \operatorname{sech}^{2} \pi p \\
\sum_{n=1}\left(e^{-2 \pi p}\right)^{|2 n+1|} & =\frac{2 e^{-2 \pi p}}{1-e^{-4 \pi p}}=\operatorname{csch} 2 \pi p
\end{aligned}
$$

for the antiperiodic case. For both cases we have been able to reduce the result to one single summation. The final step is to rewrite these sums in terms of known ones. For this purpose we rely on identities such as:

$$
\frac{1}{2 \pi} \sum_{p=1}^{\infty} \frac{\operatorname{coth} \pi p}{p^{3}}=\frac{7 \pi^{2}}{360}
$$

which can be found in [96]. We have checked numerically the validity of the identities we use with arbitrary precision, but we could not find a reference in the literature where they were proven. The massless Casimir function for periodic boundary conditions gives:

$$
\begin{aligned}
V_{\mathcal{C}}[a, 0] & =\frac{1}{(2 \pi a)^{2}}\left[\frac{1}{\pi^{2}} \sum_{p=1}^{\infty} \frac{1}{p^{4}}+\frac{1}{2 \pi} \sum_{n=-\infty}^{\infty}\left\{2 \pi|n| \operatorname{Li}_{2}\left(e^{-2 \pi|n|}\right)+\operatorname{Li}_{3}\left(e^{-2 \pi|n|}\right)\right\}\right] \\
& =\frac{1}{(2 \pi a)^{2}}\left[\frac{1}{\pi^{2}} \operatorname{Li}_{4}(1)+\frac{1}{2 \pi} \sum_{n=-\infty}^{\infty} \sum_{p=1}^{\infty}\left\{\frac{2 \pi}{p^{2}}|n|\left(e^{-2 \pi p}\right)^{|n|}+\frac{1}{p^{3}}\left(e^{-2 \pi p}\right)^{|n|}\right\}\right] \\
& =\frac{1}{(2 \pi a)^{2}}\left[\frac{\pi^{2}}{90}+\frac{1}{2 \pi} \sum_{p=1}^{\infty}\left\{2 \pi \frac{1}{2 p^{2} \sinh ^{2} \pi p}+\frac{\operatorname{coth} \pi p}{p^{3}}\right\}\right]
\end{aligned}
$$




$$
\begin{aligned}
& =\frac{1}{(2 \pi a)^{2}}\left[\frac{\pi^{2}}{90}+\frac{1}{\pi} \sum_{p=1}^{\infty}\left\{2 \pi \frac{1}{p^{2}(\cosh \pi p-1)}\right\}+\frac{7 \pi^{2}}{360}\right] \\
& =\frac{1}{(2 \pi a)^{2}} \frac{\mathcal{G}}{3},
\end{aligned}
$$

where $\mathcal{G} \simeq 0.915966$ is Catalan's constant. For the case of antiperiodic boundary conditions the Casimir energy reads:

$$
\begin{aligned}
V_{\mathcal{C}}\left[a, \frac{1}{2}\right] & =\frac{1}{(2 \pi a)^{2}}\left[\frac{1}{\pi^{2}} \sum_{p=1}^{\infty} \frac{(-1)^{p}}{p^{4}}+\frac{1}{2 \pi} \sum_{n=-\infty}^{\infty}\left\{\pi|2 n+1| \operatorname{Li}_{2}\left(-e^{-\pi|2 n+1|}\right)+\operatorname{Li}_{3}\left(-e^{-\pi|2 n+1|}\right)\right\}\right] \\
& =\frac{1}{(2 \pi a)^{2}}\left[\frac{1}{\pi^{2}} \operatorname{Li}_{4}(-1)+\frac{1}{2 \pi} \sum_{n=1}^{\infty}\left\{\frac{(-1)^{p} \pi}{p^{2}}|2 n+1|\left(e^{-\pi p}\right)^{|2 n+1|}+\frac{(-1)^{p}}{p^{3}}\left(e^{-\pi p}\right)^{|2 n+1|}\right\}\right] \\
& =\frac{1}{(2 \pi a)^{2}}\left[-\frac{7}{8} \frac{\pi^{2}}{90}-\frac{1}{2 \pi} \sum_{p=1}^{\infty}\left\{2 \pi \frac{\frac{(-1)^{p}}{4} \operatorname{csch}^{2} \pi p+\frac{(-1)^{p}}{4} \operatorname{sech}^{2} \pi p}{2 p^{2}}+\frac{(-1)^{p} \operatorname{csch} 2 \pi p}{p^{3}}\right\}\right] \\
& =\frac{-1}{(2 \pi a)^{2}} \frac{\mathcal{G}}{6}=-\frac{1}{2} V_{\mathcal{C}}[a, 0] .
\end{aligned}
$$

In the ultraviolet region of supersymmetric models, all particles are essentially massless, so the sign of the total casimir potential is determined by the next order in the expansion. Retaining the next order in the polylogarithms we find the following cuadratic terms in $a m$. For the periodic case we have:

$$
\begin{aligned}
V_{\mathcal{C}}^{(2)}[a, m, 0] & =(a m)^{2}\left\{2 \pi\left(\log (2 \pi m a)-\frac{1}{4}\right)-\operatorname{Li}_{2}(1)+\sum_{n=1}^{\infty} 2 \pi \log \left(1-e^{-2 \pi n}\right)\right\} \\
& =(a m)^{2}\left\{\pi \log (2 \pi m a)-\frac{\pi^{2}}{6}-\frac{5 \pi}{2}+\log \frac{\Gamma\left(\frac{1}{4}\right)}{2 \pi^{3 / 4}}\right\}
\end{aligned}
$$

Finally, for the antiperiodic case we find:

$$
\begin{aligned}
V_{\mathcal{C}}^{(2)}\left[a, m, \frac{1}{2}\right] & =(a m)^{2}\left\{-\operatorname{Li}_{2}(-1)+\sum_{n=-\infty}^{\infty} \pi \log \left(1+e^{-\pi|2 n+1|}\right)\right\} \\
& =(a m)^{2}\left\{-\frac{\pi^{2}}{12}+\frac{3 \pi}{4} \log 2\right\}
\end{aligned}
$$

The sign of the second order terms is opposite to the leading order sign. This means that it is positive for bosons and negative for fermions. The sign of the potential is controlled by the most massive particles in the spectra. If the fermionic degrees of freedom win, the potential will be negative and it will develop a runaway solution. 


\section{Consistency of the expansion about flat background in the non- compact dimensions}

In this appendix, we show that taking the Minkowski metric as the background metric for the calculation of the one-loop potential of the $3 \mathrm{D}$ and $2 \mathrm{D}$ theories is a valid approximation up to the energy scales that we have studied in this paper. When computing the one-loop quantum effective action, one obtains, in the general case, extra contributions from the curvature of spacetime that take the form of quadratic terms in the Riemann tensor, the Ricci tensor and the Ricci scalar. In particular, since we want to study the vacua of the theory, we are interested in the cases in which the background metric is dS, Minkowski or AdS. The typical energy scale associated to these terms is then given by the inverse of the radius of curvature, that is, the $\mathrm{dS}$ or AdS length. Hence, neglecting them (i.e. taking the background metric to be Minkowski) is justified as long as this energy scale stays well below the energy scales of the other contributions to the potential. In our case, the typical energy scale associated with the effective potential is the KK scale of the compactification and in this way we can say that our calculations are consistent and meaningful as long as we fulfill the condition

$$
l_{\mathrm{AdS} / \mathrm{dS}} \gg l_{K K}
$$

Note that it is by no means the intention of this appendix to give a detailed calculation of the contributions to the one-loop effective action coming from the non-zero curvature of the background metric but just to show that they are negligible for the cases we have studied. Before going to the two cases that are relevant for us, let us recall the general relation between the Ricci scalar and the radius of curvature (i.e. the dS or AdS length), $l$, which takes the form

$$
\mathcal{R}_{(d)}= \pm \frac{d(d-1)}{l^{2}},
$$

where the positive sign corresponds to dS whereas the negative one corresponds to AdS.

3D potential. In the circle compactification, we can use Einstein's equations to relate the Ricci scalar with the 3D cosmoical constant, $\Lambda_{(3)}$, which at the same time is related with the potential, $V$, obtaining

$$
\mathcal{R}_{(3)}=6 \Lambda_{(3)}=6 \frac{V}{M_{p}^{(3)}},
$$

where $M_{p}^{(3)}=\sqrt{2 \pi R} M_{p}$ is the $3 \mathrm{D}$ reduced Planck mass. In this way, since both the Planck mass and the potential depend on the radion, we can use eq. (C.2) to obtain an expression for $l$ as a function of the radion field and from there work out which values of $R$ fulfill the condition (C.1). This expression takes the form

$$
l=\frac{\sqrt{2 \pi R} M_{p}^{(4)}}{V^{1 / 2}} .
$$

In particular, it is interesting to rewrite this equation in terms of quantities that we can easily obtain from our plots. For that purpose we recall that in our figures we plot $R^{6} V$ 
in units of degrees of freedom, so $n=720 \pi R^{6} V$, the effective degrees of freedom, is the quantity that we read from the plots. In terms of these variables, eq. (C.4) can be reexpressed as

$$
l=\sqrt{\frac{720 \pi \times 2 \pi}{n r^{3}}} M_{p}^{(4)} R^{7 / 2},
$$

where, $r$ is just an arbitrary energy scale that ensures the right units for the potential, so we can take it to be $1 \mathrm{GeV}$ without loss of generality. From the figures we can safely say that, in these units, the potential takes values from $n=10$ to $n=70$ in all the cases we have studied (see figures 1-5), so we can take it to be $n \sim 100$ to be safe. After plugging in the numbers, one obtains that taking Minkowski as the 3D background metric in the one-loop quantum effective action calculation is justified as long as

$$
R^{-1} \ll 10^{7} \mathrm{GeV},
$$

as in all our models.

2D potential. Due to conformal invariance of $2 \mathrm{D}$ gravity, we have seen that we cannot go to the Einstein frame in order to define a canonical potential or cosmological constant in 2D. However, the equations of motion for the $T^{2} / Z_{4}$ orbifold allow us to express the $2 \mathrm{D}$ Ricci scalar in terms of the potential as follows

$$
\mathcal{R}_{(2)}=\frac{1}{\left(2 \pi M_{p}\right)^{2}} \frac{\partial_{a} V}{a} .
$$

As before, we can use eq. (C.2) to obtain a relation between the curvature radius and the potential and it takes the form

$$
l=2^{3 / 2} \pi M_{p}\left(\frac{a}{\partial_{a} V}\right) .
$$

Following the same reasoning as in the $3 \mathrm{D}$ case, we want to express this in terms of variables that we can easily read from our plots, that is, in terms of $n=12 \pi^{2} a^{2} V / \mathcal{G}$ and its derivatives with respect to $\log \left(a^{-1}[\mathrm{GeV}]\right)$. Taking all this into account, we can rewrite the previous expression as

$$
l^{2}=\frac{8 \pi^{2} M_{p}^{2}}{\log e} \frac{\mathcal{G}}{12 \pi^{2}} \frac{a^{4}}{2 a^{2} V+\partial_{\log \left(a^{-1}\right)}\left(a^{2} V\right)} .
$$

From figures $6-9$ it can be seen that $n \sim 100$ is again a safe value and that the slopes can also be overestimated by taking them to be $\sim 100$. Plugging in these values, one obtains that 2D Minkowski background is a safe approximation if we fulfill

$$
a^{-1} \ll 10^{18} \mathrm{GeV} .
$$

This again implies that, with a conservative estimation, the potential we have considered would be changed so slightly by the corrections coming from a non-vanishing $2 \mathrm{D}$ curvature that we can safely ignore them. 


\section{Example of MSSM spectrum}

Here we just show a table with the choice of SUSY masses taken to construct the figures related to the SUSY case in the text. This particular choice is taken from [97]. Note that the general results depend very weakly on the choice of spectrum.

\begin{tabular}{|c|c|c|}
\hline Particle & Mass $(\mathrm{TeV})$ & $(-1)^{\left(2 s_{p}+1\right)} n_{p}$ \\
\hline$\tilde{g}$ (gluinos) & 3.013 & +16 \\
\hline$\tilde{G}$ (gravitino) & $0.3-1$ & 2 \\
\hline$\tilde{u}_{L}, \tilde{d}_{L}, \tilde{s}_{L}, \tilde{c}_{L}$ & 2.876 & -24 \\
\hline$\tilde{u_{R}}, \tilde{d}_{R}, \tilde{s}_{R}, \tilde{c}_{R}$ & 2.76 & -24 \\
\hline$\tilde{t}_{L}$ & 2. & -6 \\
\hline$\tilde{b}_{L}$ & 2.3 & -6 \\
\hline$\tilde{t}_{R}, \tilde{b}_{R}$ & 2.4 & -12 \\
\hline$\tilde{L}_{L}$ & 1.3 & $-2 \times 5$ \\
\hline$\tilde{L}_{R}$ & 1.119 & $-2 \times 5$ \\
\hline$\tilde{\tau}_{L}$ & 0.65 & -2 \\
\hline$\tilde{\tau}_{R}$ & 1.2 & -2 \\
\hline$\chi_{1}^{0}$ & 0.55 & 2 \\
\hline$\chi_{2}^{0}$ & 1.153 & 2 \\
\hline$\chi_{3}^{0}, \chi_{4}^{0}$ & 1.9 & 4 \\
\hline$\chi_{1}^{+}$ & 1.153 & 4 \\
\hline$\chi_{2}^{+}$ & 1.9 & 4 \\
\hline$A^{0}$ & 1.592 & -1 \\
\hline $\mathcal{H}$ & 1.6 & -1 \\
\hline $\mathcal{H}^{+}$ & 1.6 & -2 \\
\hline
\end{tabular}

Table 3. Example of SUSY spectrum taken from [97].

Open Access. This article is distributed under the terms of the Creative Commons Attribution License (CC-BY 4.0), which permits any use, distribution and reproduction in any medium, provided the original author(s) and source are credited.

\section{References}

[1] C. Vafa, The string landscape and the swampland, hep-th/0509212 [INSPIRE].

[2] N. Arkani-Hamed, L. Motl, A. Nicolis and C. Vafa, The string landscape, black holes and gravity as the weakest force, JHEP 06 (2007) 060 [hep-th/0601001] [INSPIRE].

[3] H. Ooguri and C. Vafa, On the Geometry of the String Landscape and the Swampland, Nucl. Phys. B 766 (2007) 21 [hep-th/0605264] [INSPIRE]. 
[4] T. Rudelius, Constraints on Axion Inflation from the Weak Gravity Conjecture, JCAP 09 (2015) 020 [arXiv : 1503.00795] [INSPIRE].

[5] M. Montero, A.M. Uranga and I. Valenzuela, Transplanckian axions!?, JHEP 08 (2015) 032 [arXiv: 1503.03886] [INSPIRE].

[6] J. Brown, W. Cottrell, G. Shiu and P. Soler, Fencing in the Swampland: Quantum Gravity Constraints on Large Field Inflation, JHEP 10 (2015) 023 [arXiv:1503.04783] [INSPIRE].

[7] J. Brown, W. Cottrell, G. Shiu and P. Soler, On Axionic Field Ranges, Loopholes and the Weak Gravity Conjecture, JHEP 04 (2016) 017 [arXiv: 1504.00659] [INSPIRE].

[8] B. Heidenreich, M. Reece and T. Rudelius, Weak Gravity Strongly Constrains Large-Field Axion Inflation, JHEP 12 (2015) 108 [arXiv: 1506.03447] [INSPIRE].

[9] C. Cheung and G.N. Remmen, Naturalness and the Weak Gravity Conjecture, Phys. Rev. Lett. 113 (2014) 051601 [arXiv: 1402.2287] [INSPIRE].

[10] A. de la Fuente, P. Saraswat and R. Sundrum, Natural Inflation and Quantum Gravity, Phys. Rev. Lett. 114 (2015) 151303 [arXiv:1412.3457] [InSPIRE].

[11] A. Hebecker, P. Mangat, F. Rompineve and L.T. Witkowski, Winding out of the Swamp: Evading the Weak Gravity Conjecture with F-term Winding Inflation?, Phys. Lett. B 748 (2015) 455 [arXiv: 1503.07912] [INSPIRE].

[12] T.C. Bachlechner, C. Long and L. McAllister, Planckian Axions and the Weak Gravity Conjecture, JHEP 01 (2016) 091 [arXiv: 1503. 07853] [INSPIRE].

[13] T. Rudelius, On the Possibility of Large Axion Moduli Spaces, JCAP 04 (2015) 049 [arXiv: 1409.5793] [INSPIRE].

[14] D. Junghans, Large-Field Inflation with Multiple Axions and the Weak Gravity Conjecture, JHEP 02 (2016) 128 [arXiv: 1504.03566] [INSPIRE].

[15] K. Kooner, S. Parameswaran and I. Zavala, Warping the Weak Gravity Conjecture, Phys. Lett. B 759 (2016) 402 [arXiv: 1509.07049] [INSPIRE].

[16] D. Harlow, Wormholes, Emergent Gauge Fields and the Weak Gravity Conjecture, JHEP 01 (2016) 122 [arXiv: 1510.07911] [INSPIRE].

[17] L.E. Ibáñez, M. Montero, A. Uranga and I. Valenzuela, Relaxion Monodromy and the Weak Gravity Conjecture, JHEP 04 (2016) 020 [arXiv:1512.00025] [INSPIRE].

[18] A. Hebecker, F. Rompineve and A. Westphal, Axion Monodromy and the Weak Gravity Conjecture, JHEP 04 (2016) 157 [arXiv: 1512.03768] [INSPIRE].

[19] B. Heidenreich, M. Reece and T. Rudelius, Evidence for a sublattice weak gravity conjecture, JHEP 08 (2017) 025 [arXiv:1606.08437] [INSPIRE].

[20] M. Montero, G. Shiu and P. Soler, The Weak Gravity Conjecture in three dimensions, JHEP 10 (2016) 159 [arXiv:1606.08438] [INSPIRE].

[21] P. Saraswat, Weak gravity conjecture and effective field theory, Phys. Rev. D 95 (2017) 025013 [arXiv: 1608.06951] [INSPIRE].

[22] D. Klaewer and E. Palti, Super-Planckian Spatial Field Variations and Quantum Gravity, JHEP 01 (2017) 088 [arXiv: 1610.00010] [INSPIRE].

[23] L. McAllister, P. Schwaller, G. Servant, J. Stout and A. Westphal, Runaway Relaxion Monodromy, JHEP 02 (2018) 124 [arXiv:1610.05320] [INSPIRE]. 
[24] A. Herráez and L.E. Ibáñez, An Axion-induced SM/MSSM Higgs Landscape and the Weak Gravity Conjecture, JHEP 02 (2017) 109 [arXiv:1610.08836] [INSPIRE].

[25] M. Montero, Are tiny gauge couplings out of the Swampland?, JHEP 10 (2017) 208 [arXiv: 1708.02249] [INSPIRE].

[26] L.E. Ibáñez and M. Montero, A Note on the WGC, Effective Field Theory and Clockwork within String Theory, JHEP 02 (2018) 057 [arXiv:1709.02392] [INSPIRE].

[27] C. Cheung, J. Liu and G.N. Remmen, Proof of the Weak Gravity Conjecture from Black Hole Entropy, arXiv:1801.08546 [INSPIRE].

[28] T.W. Grimm, E. Palti and I. Valenzuela, Infinite Distances in Field Space and Massless Towers of States, arXiv:1802.08264 [INSPIRE].

[29] B. Heidenreich, M. Reece and T. Rudelius, Emergence and the Swampland Conjectures, arXiv: 1802.08698 [INSPIRE].

[30] S. Andriolo, D. Junghans, T. Noumi and G. Shiu, A Tower Weak Gravity Conjecture from Infrared Consistency, arXiv: 1802.04287 [INSPIRE].

[31] R. Blumenhagen, D. Kläwer, L. Schlechter and F. Wolf, The Refined Swampland Distance Conjecture in Calabi-Yau Moduli Spaces, arXiv:1803.04989 [INSPIRE].

[32] T.D. Brennan, F. Carta and C. Vafa, The String Landscape, the Swampland and the Missing Corner, arXiv:1711.00864 [INSPIRE].

[33] H. Ooguri and C. Vafa, Non-supersymmetric AdS and the Swampland, Adv. Theor. Math. Phys. 21 (2017) 1787 [arXiv:1610.01533] [INSPIRE].

[34] U. Danielsson and G. Dibitetto, Fate of stringy AdS vacua and the weak gravity conjecture, Phys. Rev. D 96 (2017) 026020 [arXiv:1611.01395] [INSPIRE].

[35] B. Freivogel and M. Kleban, Vacua Morghulis, arXiv:1610.04564 [INSPIRE].

[36] T. Banks, Note on a Paper by Ooguri and Vafa, arXiv:1611.08953 [InSPIRE].

[37] H. Ooguri and L. Spodyneiko, New Kaluza-Klein instantons and the decay of AdS vacua, Phys. Rev. D 96 (2017) 026016 [arXiv:1703.03105] [INSPIRE].

[38] S. Giombi, Higher Spin - CFT Duality, in Proceedings, Theoretical Advanced Study Institute in Elementary Particle Physics: New Frontiers in Fields and Strings (TASI 2015): Boulder, CO, U.S.A., June 1-26, 2015, pp. 137-214, arXiv:1607.02967 [INSPIRE].

[39] L.E. Ibáñez, V. Martin-Lozano and I. Valenzuela, Constraining Neutrino Masses, the Cosmological Constant and BSM Physics from the Weak Gravity Conjecture, JHEP 11 (2017) 066 [arXiv: 1706.05392] [INSPIRE].

[40] L.E. Ibáñez, V. Martin-Lozano and I. Valenzuela, Constraining the EW Hierarchy from the Weak Gravity Conjecture, arXiv:1707.05811 [INSPIRE].

[41] N. Arkani-Hamed, S. Dubovsky, A. Nicolis and G. Villadoro, Quantum Horizons of the Standard Model Landscape, JHEP 06 (2007) 078 [hep-th/0703067] [INSPIRE].

[42] Y. Hamada and G. Shiu, Weak Gravity Conjecture, Multiple Point Principle and the Standard Model Landscape, JHEP 11 (2017) 043 [arXiv: 1707.06326] [INSPIRE].

[43] Particle Data Group collaboration, C. Patrignani et al., Review of Particle Physics, Chin. Phys. C 40 (2016) 100001 [nNSPIRE]. 
[44] M. Tegmark, A. Aguirre, M. Rees and F. Wilczek, Dimensionless constants, cosmology and other dark matters, Phys. Rev. D 73 (2006) 023505 [astro-ph/0511774] [INSPIRE].

[45] L. Pogosian and A. Vilenkin, Anthropic predictions for vacuum energy and neutrino masses in the light of WMAP-3, JCAP 01 (2007) 025 [astro-ph/0611573] [INSPIRE].

[46] L.E. Ibáñez and A.M. Uranga, Neutrino Majorana Masses from String Theory Instanton Effects, JHEP 03 (2007) 052 [hep-th/0609213] [INSPIRE].

[47] R. Blumenhagen, M. Cvetič and T. Weigand, Spacetime instanton corrections in $4 D$ string vacua: The seesaw mechanism for D-brane models, Nucl. Phys. B 771 (2007) 113 [hep-th/0609191] [INSPIRE].

[48] L.E. Ibáñez, A.N. Schellekens and A.M. Uranga, Instanton Induced Neutrino Majorana Masses in CFT Orientifolds with MSSM-like spectra, JHEP 06 (2007) 011 [arXiv: 0704.1079] [INSPIRE].

[49] T. Appelquist and A. Chodos, The Quantum Dynamics of Kaluza-Klein Theories, Phys. Rev. D 28 (1983) 772 [INSPIRE].

[50] E. Ponton and E. Poppitz, Casimir energy and radius stabilization in five-dimensional orbifolds and six-dimensional orbifolds, JHEP 06 (2001) 019 [hep-ph/0105021] [INSPIRE].

[51] E. Witten, Instability of the Kaluza-Klein Vacuum, Nucl. Phys. B 195 (1982) 481 [INSPIRE].

[52] N. Cabibbo, L. Maiani, G. Parisi and R. Petronzio, Bounds on the Fermions and Higgs Boson Masses in Grand Unified Theories, Nucl. Phys. B 158 (1979) 295 [inSPIRE].

[53] J.A. Casas, J.R. Espinosa and M. Quirós, Improved Higgs mass stability bound in the standard model and implications for supersymmetry, Phys. Lett. B 342 (1995) 171 [hep-ph/9409458] [INSPIRE].

[54] G. Altarelli and G. Isidori, Lower limit on the Higgs mass in the standard model: An update, Phys. Lett. B 337 (1994) 141 [inSPIRE].

[55] J.A. Casas, J.R. Espinosa and M. Quirós, Standard model stability bounds for new physics within LHC reach, Phys. Lett. B 382 (1996) 374 [hep-ph/9603227] [INSPIRE].

[56] J. Elias-Miro, J.R. Espinosa, G.F. Giudice, G. Isidori, A. Riotto and A. Strumia, Higgs mass implications on the stability of the electroweak vacuum, Phys. Lett. B 709 (2012) 222 [arXiv:1112.3022] [INSPIRE].

[57] G. Degrassi et al., Higgs mass and vacuum stability in the Standard Model at NNLO, JHEP 08 (2012) 098 [arXiv: 1205.6497] [INSPIRE].

[58] L.E. Ibáñez and I. Valenzuela, The Higgs Mass as a Signature of Heavy SUSY, JHEP 05 (2013) 064 [arXiv:1301.5167] [INSPIRE].

[59] L.E. Ibáñez, F. Marchesano, D. Regalado and I. Valenzuela, The Intermediate Scale MSSM, the Higgs Mass and F-theory Unification, JHEP 07 (2012) 195 [arXiv:1206.2655] [INSPIRE].

[60] J.M. Arnold, B. Fornal and M.B. Wise, Standard Model Vacua for Two-dimensional Compactifications, JHEP 12 (2010) 083 [arXiv: 1010.4302] [INSPIRE].

[61] J.M. Arnold, B. Fornal and K. Ishiwata, Finite Temperature Structure of the Compactified Standard Model, JHEP 08 (2011) 030 [arXiv:1103.0002] [INSPIRE].

[62] D.M. Ghilencea, D. Hoover, C.P. Burgess and F. Quevedo, Casimir energies for $6 D$ supergravities compactified on $T_{2} / Z_{N}$ with Wilson lines, JHEP 09 (2005) 050 [hep-th/0506164] [INSPIRE]. 
[63] L.E. Ibáñez, A.N. Schellekens and A.M. Uranga, Discrete Gauge Symmetries in Discrete MSSM-like Orientifolds, Nucl. Phys. B 865 (2012) 509 [arXiv:1205.5364] [InSPIRE].

[64] M. Berasaluce-Gonzalez, L.E. Ibáñez, P. Soler and A.M. Uranga, Discrete gauge symmetries in D-brane models, JHEP 12 (2011) 113 [arXiv:1106.4169] [INSPIRE].

[65] A. Font, L.E. Ibáñez, F. Quevedo and A. Sierra, The Construction of 'Realistic' Four-Dimensional Strings Through Orbifolds, Nucl. Phys. B 331 (1990) 421 [INSPIRE].

[66] H.P. Nilles, Stringy Origin of Discrete R-symmetries, PoS (CORFU2016) 017 [arXiv: 1705. 01798] [INSPIRE].

[67] T. Kobayashi, H.P. Nilles, F. Ploger, S. Raby and M. Ratz, Stringy origin of non-Abelian discrete flavor symmetries, Nucl. Phys. B 768 (2007) 135 [hep-ph/0611020] [INSPIRE].

[68] H.M. Lee et al., Discrete R symmetries for the MSSM and its singlet extensions, Nucl. Phys. B 850 (2011) 1 [arXiv:1102.3595] [INSPIRE].

[69] M. Berasaluce-Gonzalez, P.G. Camara, F. Marchesano, D. Regalado and A.M. Uranga, Non-Abelian discrete gauge symmetries in 4d string models, JHEP 09 (2012) 059 [arXiv: 1206.2383] [INSPIRE].

[70] P. Anastasopoulos, R. Richter and A.N. Schellekens, Discrete symmetries from hidden sectors, JHEP 06 (2015) 189 [arXiv: 1502.02686] [INSPIRE].

[71] T.P.T. Dijkstra, L.R. Huiszoon and A.N. Schellekens, Supersymmetric standard model spectra from RCFT orientifolds, Nucl. Phys. B 710 (2005) 3 [hep-th/0411129] [INSPIRE].

[72] B. Gato-Rivera and A.N. Schellekens, Asymmetric Gepner Models III. B-L Lifting, Nucl. Phys. B 847 (2011) 532 [arXiv:1012.0796] [InSPIRE].

[73] G. Honecker and W. Staessens, To Tilt or Not To Tilt: Discrete Gauge Symmetries in Global Intersecting D-brane Models, JHEP 10 (2013) 146 [arXiv:1303.4415] [INSPIRE].

[74] J. Ecker, G. Honecker and W. Staessens, D6-brane model building on $\mathbb{Z}_{2} \times \mathbb{Z}_{6}$ : MSSM-like and left-right symmetric models, Nucl. Phys. B 901 (2015) 139 [arXiv:1509.00048] [INSPIRE].

[75] J.P. Derendinger, S. Ferrara, C. Kounnas and F. Zwirner, On loop corrections to string effective field theories: Field dependent gauge couplings and $\sigma$-model anomalies, Nucl. Phys. B 372 (1992) 145 [INSPIRE].

[76] G. Lopes Cardoso and B.A. Ovrut, A Green-Schwarz mechanism for $D=4, N=1$ supergravity anomalies, Nucl. Phys. B 369 (1992) 351 [INSPIRE].

[77] G. Lopes Cardoso and B.A. Ovrut, Coordinate and Kähler $\sigma$-model anomalies and their cancellation in string effective field theories, Nucl. Phys. B 392 (1993) 315 [hep-th/9205009] [INSPIRE].

[78] L.E. Ibáñez and D. Lüst, Duality anomaly cancellation, minimal string unification and the effective low-energy Lagrangian of 4-D strings, Nucl. Phys. B 382 (1992) 305 [hep-th/9202046] [INSPIRE].

[79] L.E. Ibáñez, R. Rabadán and A.M. Uranga, $\sigma$-model anomalies in compact $D=4, N=1$ type IIB orientifolds and Fayet-Iliopoulos terms, Nucl. Phys. B 576 (2000) 285 [hep-th/9905098] [INSPIRE].

[80] L.E. Ibáñez and G.G. Ross, Discrete gauge symmetry anomalies, Phys. Lett. B 260 (1991) 291 [INSPIRE]. 
[81] L.E. Ibáñez and G.G. Ross, Discrete gauge symmetries and the origin of baryon and lepton number conservation in supersymmetric versions of the standard model, Nucl. Phys. B 368 (1992) 3 [INSPIRE].

[82] T. Banks and M. Dine, Note on discrete gauge anomalies, Phys. Rev. D 45 (1992) 1424 [hep-th/9109045] [INSPIRE].

[83] L.E. Ibáñez, More about discrete gauge anomalies, Nucl. Phys. B 398 (1993) 301 [hep-ph/9210211] [INSPIRE].

[84] J.M. Frere, D.R.T. Jones and S. Raby, Fermion Masses and Induction of the Weak Scale by Supergravity, Nucl. Phys. B 222 (1983) 11 [INSPIRE].

[85] J.A. Casas, A. Lleyda and C. Muñoz, Strong constraints on the parameter space of the MSSM from charge and color breaking minima, Nucl. Phys. B 471 (1996) 3 [hep-ph/9507294] [INSPIRE].

[86] W.G. Hollik, A new view on vacuum stability in the MSSM, JHEP 08 (2016) 126 [arXiv: 1606.08356] [INSPIRE].

[87] A. Font and J.A. Lopez, Strings on eight-orbifolds, Nucl. Phys. B 703 (2004) 177 [hep-th/0405151] [INSPIRE].

[88] S. Sethi, C. Vafa and E. Witten, Constraints on low dimensional string compactifications, Nucl. Phys. B 480 (1996) 213 [hep-th/9606122] [INSPIRE].

[89] K. Dasgupta and S. Mukhi, A note on low dimensional string compactifications, Phys. Lett. B 398 (1997) 285 [hep-th/9612188] [INSPIRE].

[90] S. Förste and D. Ghoshal, Strings from orientifolds, Nucl. Phys. B 527 (1998) 95 [hep-th/9711039] [INSPIRE].

[91] S. Schäfer-Nameki and T. Weigand, F-theory and 2d (0,2) theories, JHEP 05 (2016) 059 [arXiv: 1601.02015] [INSPIRE].

[92] F. Apruzzi, F. Hassler, J.J. Heckman and I.V. Melnikov, UV Completions for Non-Critical Strings, JHEP 07 (2016) 045 [arXiv: 1602.04221] [INSPIRE].

[93] T. Weigand and F. Xu, The Green-Schwarz Mechanism and Geometric Anomaly Relations in 2d (0,2) F-theory Vacua, JHEP 04 (2018) 107 [arXiv:1712.04456] [InSPIRE].

[94] L. Álvarez-Gaumé and E. Witten, Gravitational Anomalies, Nucl. Phys. B 234 (1984) 269 [INSPIRE].

[95] L. Lewin, Polylogarithms and Associated Functions, Elsevier Science Ltd, (1981).

[96] B.C. Berndt, Ramanujan Notebooks Part II, chapter 14, eq. (25.1), Springer (1989)

[97] L. Aparicio, D.G. Cerdeño and L.E. Ibáñez, A 119-125 GeV Higgs from a string derived slice of the CMSSM, JHEP 04 (2012) 126 [arXiv:1202.0822] [INSPIRE]. 